ESAIM: M2AN

Vol. 40, No 1, 2006, pp. 175-199

DOI: $10.1051 / \mathrm{m} 2 \mathrm{an}: 2006005$
ESAIM: Mathematical Modelling and Numerical Analysis

www.edpsciences.org/m2an

\title{
CONVERGENCE OF A FULLY DISCRETE FINITE ELEMENT METHOD FOR A DEGENERATE PARABOLIC SYSTEM MODELLING NEMATIC LIQUID CRYSTALS WITH VARIABLE DEGREE OF ORIENTATION*
}

\author{
John W. Barrett ${ }^{1}$, Xiaobing Feng ${ }^{2}$ And Andreas Prohl ${ }^{3}$
}

\begin{abstract}
We consider a degenerate parabolic system which models the evolution of nematic liquid crystal with variable degree of orientation. The system is a slight modification to that proposed in [Calderer et al., SIAM J. Math. Anal. 33 (2002) 1033-1047], which is a special case of Ericksen's general continuum model in [Ericksen, Arch. Ration. Mech. Anal. 113 (1991) 97-120]. We prove the global existence of weak solutions by passing to the limit in a regularized system. Moreover, we propose a practical fully discrete finite element method for this regularized system, and we establish the (subsequence) convergence of this finite element approximation to the solution of the regularized system as the mesh parameters tend to zero; and to a solution of the original degenerate parabolic system when the the mesh and regularization parameters all approach zero. Finally, numerical experiments are included which show the formation, annihilation and evolution of line singularities/defects in such models.
\end{abstract}

Mathematics Subject Classification. 35K55, 35K65, 35Q35, 65M12, 65M60, 76A15.

Received: January 31, 2005. Revised: September 30, 2005.

\section{INTRODUCTION}

Let $\Omega$ be a bounded domain in $\mathbb{R}^{d}, d \leq 3$. In this paper we consider, for any given constants $k_{i}>0, \delta \geq 0$ and $p>d$, the degenerate parabolic system:

$\left(\mathbf{P}_{\delta}\right)$ Find $\{s, \varphi\}$ such that

$$
\begin{aligned}
\partial_{t} s & =k_{1} \operatorname{div}\left(\left[1+\delta|\nabla s|^{p-2}\right] \nabla s\right)-k_{2}|\nabla \varphi|^{2} s-W^{\prime}(s) & & \text { in } \quad \Omega_{T}:=\Omega \times(0, T), \quad 0<T<\infty, \\
s^{2} \partial_{t} \varphi & =k_{3} \operatorname{div}\left(s^{2} \nabla \varphi\right) & & \text { in } \quad \Omega_{T}
\end{aligned}
$$

subject to appropriate initial and boundary conditions. The system $\left(\mathrm{P}_{\delta}\right)$ models the evolution of uniaxial nematic liquid crystals with variable degree of orientation in the absence of both flow and electromagnetic fields. The model $\left(\mathrm{P}_{0}\right)$; that is, $\left(\mathrm{P}_{\delta}\right)$ with $\delta=0$; was derived by Calderer, Golovaty, Lin and Liu in [2] and is

Keywords and phrases. Nematic liquid crystal, degenerate parabolic system, existence, finite element method, convergence.

* Part of this work was carried out while the authors participated in the 2003 programme Computational Challenges in Partial Differential Equations at the Isaac Newton Institute, Cambridge, UK.

1 Department of Mathematics, Imperial College, London, SW7 2AZ, UK. jwb@ic.ac.uk

2 Department of Mathematics, The University of Tennessee, Knoxville, TN 37996, USA.

3 Department of Mathematics, ETH, 8092 Zürich, Switzerland.

(C) EDP Sciences, SMAI 2006 
a special case of Ericksen's general continuum theory for nematic polymers with variable degree of orientation, which was proposed in [4]. A typical nematic liquid crystal consists of rigid, rodlike molecules with one molecular axis being much longer than the other two. Hence there is a high probability that the axes of any two neighboring molecules point in a similar direction. The local molecular orientation of a uniaxial nematic liquid crystal can be specified by a unit vector $\mathbf{n}(x, t)$, called the director, and a scalar $s(x, t)$, called an order parameter. In this continuum model, $s(x, t)$ is the averaged local orientation of molecules compared to $\mathbf{n}(x, t)$. It can be shown that $s(x, t) \in\left[-\frac{1}{2}, 1\right]$; where $s(x, t)=1\left(-\frac{1}{2}\right)$ corresponds to all molecules being locally aligned (perpendicular) to $\mathbf{n}(x, t)$; see [4] for details. The special case of $s(x, t)=0$ corresponds to the isotropic state in which the director $\mathbf{n}(x, t)$ is meaningless. Obviously, setting $s \equiv 1$ in the Ericksen model corresponds to the Leslie-Ericksen model, where the configuration is determined solely by $\mathbf{n}(x, t)$. This simplified model has been successful in describing low molar-mass nematics. However, it only gives rise to point singularities in $\mathbf{n}(x, t)$, whereas line and surface singularities are observed in laboratory experiments. Hence, Ericksen [4] introduced the extra scalar parameter $s$, which allows for variable degree of orientation, in order to overcome the deficiencies in singularity formation of the Leslie-Ericksen model for general uniaxial nematics.

The bounded domain $\Omega \subset \mathbb{R}^{d}$, for $d \leq 3$, is the region occupied by the liquid crystal material in the model $\left(\mathrm{P}_{\delta}\right)$. Whereas only planar director configurations $\mathbf{n}=(\cos \varphi, \sin \varphi, 0)$ are allowed, where $\varphi$ is the associated angle. The positive constants $k_{1}, k_{2}$ and $k_{3}$ depend on material properties. Finally, $W^{\prime}(s)$ denotes the derivative of a smooth double well potential such that $W^{\prime}\left(-\frac{1}{2}\right)<0$ and $W^{\prime}(1)>0$. This ensures that the perfect alignments $s=-\frac{1}{2}$ and $s=1$ are excluded from being equilibrium states, which is physically reasonable; see ([4], p. 109).

In order to overcome the degeneracy in (1.1b) at $s=0$, Calderer, Golovaty, Lin and Liu [2] considered a regularization $\left(\mathrm{P}_{0, \varepsilon}\right)$ of $\left(\mathrm{P}_{0}\right)$. Here we extend this regularization to $\left(\mathrm{P}_{\delta}\right)$, and consider for any given constant $\varepsilon \in(0,1]:$

$\left(\mathbf{P}_{\delta, \varepsilon}\right)$ Find $\left\{s_{\varepsilon}, \varphi_{\varepsilon}\right\}$ such that

$$
\begin{aligned}
\partial_{t} s_{\varepsilon} & =k_{1} \operatorname{div}\left(\left[1+\delta\left|\nabla s_{\varepsilon}\right|^{p-2}\right] \nabla s_{\varepsilon}\right)-k_{2}\left|\nabla \varphi_{\varepsilon}\right|^{2} s_{\varepsilon}-W^{\prime}\left(s_{\varepsilon}\right) & & \text { in } \Omega_{T}, \\
\left(s_{\varepsilon}^{2}+\varepsilon^{2}\right) \partial_{t} \varphi_{\varepsilon} & =k_{3} \operatorname{div}\left(\left(s_{\varepsilon}^{2}+\varepsilon^{2}\right) \nabla \varphi_{\varepsilon}\right) & & \text { in } \Omega_{T}
\end{aligned}
$$

subject to the same initial and boundary conditions as $\left(\mathrm{P}_{\delta}\right)$. Calderer, Golovaty, Lin and Liu [2] showed that as for the original system $\left(\mathrm{P}_{0}\right)$, the regularized system $\left(\mathrm{P}_{0, \varepsilon}\right)$ also satisfies a dissipative energy law, which in turn implies some uniform (in $\varepsilon$ ) estimates for $\left\{s_{\varepsilon}, \varphi_{\varepsilon}\right\}$. Based on these uniform estimates, they then showed global existence of weak solutions to the original system $\left(\mathrm{P}_{0}\right)$, for a smooth domain $\Omega$, using compactness arguments. However, there are a number of gaps in the proof given there for passing to the limit $\varepsilon \rightarrow 0$ in the term $\left|\nabla \varphi_{\varepsilon}\right|^{2} s_{\varepsilon}$ on the right hand side of (1.2a). Although we are able to fill most of these gaps, we are only able to fill all the gaps for $\left(\mathrm{P}_{0}\right)$ when $d=1$, and for the modified degenerate system $\left(\mathrm{P}_{\delta}\right)$, with $\delta>0$ and $p>d$, when $d \geq 2$. We note that $\left(\mathrm{P}_{\delta}\right)$ with $\delta>0$ is still a degenerate system and satisfies a similar dissipative energy law to $\left(\mathrm{P}_{0}\right)$. Whereas for $\left(\mathrm{P}_{0, \varepsilon}\right)$ when $d \geq 2$, we can only establish the strong convergence of a subsequence $s_{\varepsilon}$ to $s$ in $L^{q}\left(\Omega_{T}\right)$ for any $q \in[1, \infty)$; for $\left(\mathrm{P}_{\delta, \varepsilon}\right)$ with $\delta>0$ and $p>d$ we can establish this convergence in $L^{\infty}\left(\Omega_{T}\right)$. This plays a crucial role in the final step of passing to the limit $\varepsilon \rightarrow 0$ in $\left(\mathrm{P}_{\delta, \varepsilon}\right)$. This is the only reason for this $p$-Laplacian modification of $\left(\mathrm{P}_{0}\right)$ in the case $d \geq 2$.

The first goal of this paper is to develop a practical fully discrete finite element method, $\left(\mathrm{P}_{\delta, \varepsilon}^{h, \tau}\right)$, for approximating the solution to the regularized system $\left(\mathrm{P}_{\delta, \varepsilon}\right)$ for $\delta \geq 0$ and $p>d$, and to show (subsequence) convergence of this finite element approximation to the solution of $\left(\mathrm{P}_{\delta, \varepsilon}\right)$ as the mesh size $h$ and the time step $\tau$ tend to zero. To prove this convergence, the main step is to establish a discrete dissipative energy law for this finite element approximation. The second goal is to establish (subsequence) convergence of the finite element approximation to a global weak solution of the original system $\left(\mathrm{P}_{\delta}\right)$ as the mesh size, time step and the regularization parameter $\varepsilon$ all go to zero. To this end, we re-examine the regularized system $\left(\mathrm{P}_{\delta, \varepsilon}\right)$ and provide a detailed proof of the compactness arguments for passing to the limit, as $\varepsilon \rightarrow 0$, to establish existence of weak solutions of $\left(\mathrm{P}_{\delta}\right)$; which is far from being straightforward because of the strong coupling of the nonlinearities in the system. It is at the final stage of this process that we have to make the restrictions of $\delta>0$ and $p>d$ when $d \geq 2$. 
The paper is organized as follows. In Section 2, we first study the regularized system $\left(\mathrm{P}_{\delta, \varepsilon}\right)$ and establish well-posedness for any given $\delta \geq 0$ and $p>d$. We then prove the existence of global weak solutions for the degenerate systems $\left(\mathrm{P}_{0}\right)$ when $d=1$, and $\left(\mathrm{P}_{\delta}\right)$ with $\delta>0$ and $p>d$ when $d \geq 1$ by passing to the limit as $\varepsilon \rightarrow 0$ in the regularized system. In Section 3, we propose and analyze our fully discrete finite element method, $\left(\mathrm{P}_{\delta, \varepsilon}^{h, \tau}\right)$, for approximating solutions of both the regularized system $\left(\mathrm{P}_{\delta, \varepsilon}\right)$ and the degenerate system $\left(\mathrm{P}_{\delta}\right)$. The (subsequence) convergence of this finite element approximation is first proved for fixed $\delta \geq 0, p>d$ and $\varepsilon \in(0,1]$ as $h$ and $\tau$ go to zero; and then for a fixed (a) $\delta=0$ if $d=1$ and (b) $\delta>0$ and $p>d$ if $d \geq 1$, as $h$, $\tau$ and $\varepsilon$ go to zero. Finally, in Section 4, we present some numerical experiments to demonstrate the efficiency of the finite element method $\left(\mathrm{P}_{0, \varepsilon}^{h, \tau}\right)$.

\section{Existence of GLobal Weak solutions to the Degenerate System $\left(\mathrm{P}_{\delta}\right)$}

Global existence of weak solutions to the system $\left(\mathrm{P}_{0}\right)$ was considered in Theorem 4.3 of [2]. However, there are a number of gaps in the proof given there for passing to the limit $\varepsilon \rightarrow 0$ in the term $\left|\nabla \varphi_{\varepsilon}\right|^{2} s_{\varepsilon}$ on the right hand side of (1.2a). In this section, we will revisit the problem and present a complete proof of the existence theorem for (a) ( $\left.\mathrm{P}_{0}\right)$ if $d=1$, and (b) $\left(\mathrm{P}_{\delta}\right)$ with $\delta>0$ and $p>d$ if $d \geq 1$.

Both the system $\left(\mathrm{P}_{\delta}\right)$, for $\delta \geq 0$, and its regularized version $\left(\mathrm{P}_{\delta, \varepsilon}\right)$ must be supplemented by initial and boundary conditions in order to be a closed system. As in [2], the following initial and boundary conditions will be considered in this paper

$$
\begin{array}{lllrl}
\text { (a) } s(x, 0)=s_{\varepsilon}(x, 0)=g(x), & & \text { (b) } \varphi(x, 0)=\varphi_{\varepsilon}(x, 0)=\phi(x), & & x \in \Omega, \\
\text { (a) } s(x, t)=s_{\varepsilon}(x, t)=g(x), & & \text { (b) } \varphi(x, t)=\varphi_{\varepsilon}(x, t)=\phi(x), & & (x, t) \in \partial \Omega_{T} ;
\end{array}
$$

where $\partial \Omega_{T}:=\partial \Omega \times(0, T)$. Throughout the paper, we adopt the standard notation for Sobolev spaces and their associated norms. For notational convenience, we drop the domain from the norm and semi-norm subscript, if the domain is $\Omega$; e.g. norm $\|\cdot\|_{W^{m, r}}:=\|\cdot\|_{W^{m, r}(\Omega)}$ and semi-norm $|\cdot|_{W^{m, r}}:=|\cdot|_{W^{m, r}(\Omega)}$. We introduce also

$$
W_{g}^{1, p^{\star}}(\Omega):=\left\{v \in W^{1, p^{\star}}(\Omega): v=g \text { on } \partial \Omega\right\}, \quad H_{\phi}^{1}(\Omega):=\left\{\eta \in H^{1}(\Omega): \eta=\phi \text { on } \partial \Omega\right\} ;
$$

where $p^{\star}:=2$ if $\delta=0$ and $p^{\star}:=\max \{p, 2\}$ if $\delta>0$. Finally, throughout $(\cdot, \cdot)$ denotes the standard inner product over $L^{2}(\Omega)$.

\subsection{Well-posedness of the regularized system $\left(\mathbf{P}_{\delta, \varepsilon}\right)$}

In this subsection, we consider and analyze the regularized system $\left(\mathrm{P}_{\delta, \varepsilon}\right)$ with $\delta \geq 0$ and $p>d$ subject to the initial and boundary conditions $(2.1)$ and $(2.2)$ for a fixed $\varepsilon \in(0,1]$. We begin with definitions of weak and strong solutions of $\left(\mathrm{P}_{\delta, \varepsilon}\right)$.

Definition 2.1. Let $\Omega \subset \mathbb{R}^{d}, d \leq 3$, be a bounded domain with a Lipschitz boundary $\partial \Omega, k_{i} \in \mathbb{R}^{+}$and $W(\cdot)$ be a smooth double well potential. Assume that $g \in W^{1, p^{*}}(\Omega) \cap L^{\infty}(\Omega)$ with $g(x) \in\left[g_{-}, g_{+}\right]$for a.e. $x \in \Omega$, with $g_{-} \leq 0, g_{+} \geq 0, W^{\prime}\left(g_{-}\right) \leq 0$ and $W^{\prime}\left(g_{+}\right) \geq 0$; and that $\phi \in H^{1}(\Omega) \cap L^{\infty}(\Omega)$ with $\phi(x) \in\left[\phi_{-}, \phi_{+}\right]$for a.e. $x \in \Omega$. Then a pair of functions $\left\{s_{\varepsilon}, \varphi_{\varepsilon}\right\}$ is said to be a weak solution to $\left(\mathrm{P}_{\delta, \varepsilon}\right)$, with $\delta \geq 0, p>d$ and $\varepsilon \in(0,1]$, subject to $(2.1)$ and $(2.2)$ if $\left\{s_{\varepsilon}, \varphi_{\varepsilon}\right\}$ satisfies

(i) $s_{\varepsilon} \in H^{1}\left((0, T) ; L^{2}(\Omega)\right) \cap L^{\infty}\left((0, T) ; W_{g}^{1, p^{\star}}(\Omega)\right)$ and $\varphi_{\varepsilon} \in H^{1}\left((0, T) ; L^{2}(\Omega)\right) \cap L^{\infty}\left((0, T) ; H_{\phi}^{1}(\Omega)\right)$;

(ii) $s_{\varepsilon}(x, t) \in\left[g_{-}, g_{+}\right]$and $\varphi_{\varepsilon}(x, t) \in\left[\phi_{-}, \phi_{+}\right]$for a.e. $(x, t) \in \Omega_{T}$;

(iii) $(2.1)\left(\right.$ a) in $Y_{1}$, where $W^{1, p^{*}}(\Omega) \stackrel{c}{\hookrightarrow} Y_{1}$, and $(2.1)\left(\right.$ b) in $Y_{2}$, where $H^{1}(\Omega) \stackrel{c}{\hookrightarrow} Y_{2}$, e.g. $Y_{1}=Y_{2}=L^{2}(\Omega)$;

(iv) the following identities: 


$$
\begin{array}{rc}
\int_{\Omega_{T}}\left\{\partial_{t} s_{\varepsilon} v+k_{1}\left[1+\delta\left|\nabla s_{\varepsilon}\right|^{p-2}\right] \nabla s_{\varepsilon} \cdot \nabla v+k_{2}\left|\nabla \varphi_{\varepsilon}\right|^{2} s_{\varepsilon} v+W^{\prime}\left(s_{\varepsilon}\right) v\right\} \mathrm{d} x \mathrm{~d} t=0 & \forall v \in L^{2}\left((0, T) ; W_{0}^{1, p^{\star}}(\Omega) \cap L^{\infty}(\Omega)\right), \\
\int_{\Omega_{T}}\left\{\left(s_{\varepsilon}^{2}+\varepsilon^{2}\right) \partial_{t} \varphi_{\varepsilon} \eta+k_{3}\left(s_{\varepsilon}^{2}+\varepsilon^{2}\right) \nabla \varphi_{\varepsilon} \cdot \nabla \eta\right\} \mathrm{d} x \mathrm{~d} t=0 & \forall \eta \in L^{2}\left((0, T) ; H_{0}^{1}(\Omega)\right) .
\end{array}
$$

Remark 2.2. In the above definition, for the sake of mathematical generality, we have relaxed the physically realistic condition that $g_{-}$and $g_{+}$satisfy, in addition, $\left[g_{-}, g_{+}\right] \subset\left(-\frac{1}{2}, 1\right)$.

Definition 2.3. A weak solution $\left\{s_{\varepsilon}, \varphi_{\varepsilon}\right\}$ is called a strong solution to $\left(\mathrm{P}_{\delta, \varepsilon}\right)$ subject to (2.1) and (2.2) if $\left\{s_{\varepsilon}, \varphi_{\varepsilon}\right\} \in L^{2}\left((0, T), H^{2}(\Omega)\right)$.

Firstly in the theorem below, we prove the existence of weak solutions. In order to motivate the convergence proofs for our fully discrete finite element approximation of $\left(\mathrm{P}_{\delta, \varepsilon}\right)$ in Section 3, we consider a discrete in time/continuous in space approximation of $\left(\mathrm{P}_{\delta, \varepsilon}\right)$ here. Moreover, the desired $L^{\infty}\left(\Omega_{T}\right)$ bounds on $\left\{s_{\varepsilon}, \varphi_{\varepsilon}\right\}$ are far easier to establish using this approach.

For any $T>0$, let $0=t_{0}<t_{1}<\ldots<t_{M-1}<t_{M}=T$ be a partitioning of $[0, T]$ into possibly variable time steps $\tau_{m}:=t_{m}-t_{m-1}, m=1,2, \cdots M$. We set $\tau:=\max _{m=1,2, \cdots M} \tau_{m}$. In addition, we split the smooth double well potential $W$ into its convex and concave parts, by writing it as

$$
W(s)=W_{+}(s)-W_{-}(s), \quad \text { where } W_{+} \text {and } W_{-} \text {are convex functions. }
$$

Furthermore, for the convenience of the analysis, we introduce $\widetilde{W}(s)=\widetilde{W}_{+}(s)-\widetilde{W}_{-}(s)$, where

$$
\widetilde{W}_{( \pm)}(s):=\left\{\begin{array}{ll}
W_{( \pm)}\left(g_{-}\right)+\left(s-g_{-}\right) W_{( \pm)}^{\prime}\left(g_{-}\right) & s \leq g_{-} \\
W_{( \pm)}(s) & s \in\left[g_{-}, g_{+}\right] . \\
W_{( \pm)}\left(g_{+}\right)+\left(s-g_{+}\right) W_{( \pm)}^{\prime}\left(g_{+}\right) & s \geq g_{+}
\end{array} .\right.
$$

Here and throughout $\cdot{ }_{(\star)}$ denotes an expression with or without the subscript $\star$, similarly with superscripts.

For given $\delta \geq 0, p>d$ and $\varepsilon \in(0,1]$, we now consider the following approximation of $\left(\mathrm{P}_{\delta, \varepsilon}\right)$ :

$\left(\mathbf{P}_{\delta, \varepsilon}^{\tau}\right)$ Let $\left\{s_{\varepsilon}^{0}, \varphi_{\varepsilon}^{0}\right\}=\{g, \varphi\}$; then for $m=1,2, \cdots M$, find $s_{\varepsilon}^{m} \in V_{g}\left(\varphi_{\varepsilon}^{m-1}\right):=\left\{v \in W_{g}^{1, p^{\star}}(\Omega): v \nabla \varphi_{\varepsilon}^{m-1} \in\right.$ $\left.\left[L^{2}(\Omega)\right]^{d}\right\}$ and $\varphi_{\varepsilon}^{m} \in H_{\phi}^{1}(\Omega)$ such that

$$
\begin{aligned}
& \left(d_{t} s_{\varepsilon}^{m}, v\right)+k_{1}\left(\left[1+\delta\left|\nabla s_{\varepsilon}^{m}\right|^{p-2}\right] \nabla s_{\varepsilon}^{m}, \nabla v\right)+\left(k_{2}\left|\nabla \varphi_{\varepsilon}^{m-1}\right|^{2} s_{\varepsilon}^{m}+\widetilde{W}_{+}^{\prime}\left(s_{\varepsilon}^{m}\right), v\right)=\left(\widetilde{W}_{-}^{\prime}\left(s_{\varepsilon}^{m-1}\right), v\right) \quad \forall v \in V_{0}\left(\varphi_{\varepsilon}^{m-1}\right), \\
& \left(\left[\left(s_{\varepsilon}^{m}\right)^{2}+\varepsilon^{2}\right] d_{t} \varphi_{\varepsilon}^{m}, \eta\right)+k_{3}\left(\left[\left(s_{\varepsilon}^{m}\right)^{2}+\varepsilon^{2}\right] \nabla \varphi_{\varepsilon}^{m}, \nabla \eta\right)=0 \quad \forall \eta \in H_{0}^{1}(\Omega) ;
\end{aligned}
$$

where $d_{t} v^{m}:=\left(v^{m}-v^{m-1}\right) / \tau_{m}$.

Theorem 2.4. For any fixed $\delta \geq 0, p>d$ and $\varepsilon \in(0,1]$, the system $\left(P_{\delta, \varepsilon}\right)$ subject to $(2.1)$ and $(2.2)$ has a weak solution for all $T>0$. Moreover, its corresponding norms in (i) of Definition 2.1 are bounded independently of $\varepsilon$. Furthermore, it satisfies the following dissipative energy law for a.a. $\tilde{t} \in(0, T)$

$$
E_{\varepsilon}\left(s_{\varepsilon}, \varphi_{\varepsilon}\right)(\tilde{t})+\int_{\Omega_{\tilde{t}}}\left\{\left(\partial_{t} s_{\varepsilon}\right)^{2}+\frac{k_{2}}{k_{3}}\left(s_{\varepsilon}^{2}+\varepsilon^{2}\right)\left(\partial_{t} \varphi_{\varepsilon}\right)^{2}\right\} \mathrm{d} x \mathrm{~d} t \leq E_{\varepsilon}(g, \phi) \leq C,
$$

where $C$ is independent of $\varepsilon$ and

$$
E_{\varepsilon}\left(s_{\varepsilon}, \varphi_{\varepsilon}\right)(t):=\int_{\Omega}\left\{k_{1}\left[\frac{1}{2}\left|\nabla s_{\varepsilon}(x, t)\right|^{2}+\frac{\delta}{p}\left|\nabla s_{\varepsilon}(x, t)\right|^{p}\right]+\frac{k_{2}}{2}\left(s_{\varepsilon}^{2}(x, t)+\varepsilon^{2}\right)\left|\nabla \varphi_{\varepsilon}(x, t)\right|^{2}+W\left(s_{\varepsilon}(x, t)\right)\right\} \mathrm{d} x .
$$


Proof. For $m=1,2, \cdots M$, it is easily deduced that $V_{g}\left(\varphi_{\varepsilon}^{m-1}\right)$ is a closed convex set of the reflexive Banach space $W^{1, p^{\star}}(\Omega)$ and $(2.7 \mathrm{a})$ is the Euler-Lagrange equation associated with the minimization over $V_{g}\left(\varphi_{\varepsilon}^{m-1}\right)$ of the convex, coercive and differentiable functional

$$
J(v):=\int_{\Omega}\left\{\frac{1}{2 \tau_{m}} v^{2}+k_{1}\left[\frac{1}{2}|\nabla v|^{2}+\frac{\delta}{p}|\nabla v|^{p}\right]+\frac{k_{2}}{2}\left|\nabla \varphi_{\varepsilon}^{m-1}\right|^{2} v^{2}+\widetilde{W}_{+}(v)-\left[\frac{1}{\tau_{m}} s_{\varepsilon}^{m-1}+\widetilde{W}_{-}^{\prime}\left(s_{\varepsilon}^{m-1}\right)\right] v\right\} \mathrm{d} x
$$

Hence there exists a unique solution to (2.7a). Similarly, there exists a unique solution to (2.7b). Assuming $s_{\varepsilon}^{m-1} \in\left[g_{-}, g_{+}\right]$, and choosing $v=\left[s_{\varepsilon}^{m}-g_{+}\right]_{+} \in V_{0}\left(\varphi_{\varepsilon}^{m-1}\right)$ in (2.7a) yields, on noting the convexity of $\widetilde{W}_{ \pm}$and our assumptions on $g_{ \pm}$, that

$$
\begin{gathered}
\frac{1}{\tau_{m}}\left\|\left[s_{\varepsilon}^{m}-g_{+}\right]_{+}\right\|_{L^{2}}^{2}+k_{1}\left[\left\|\nabla\left[s_{\varepsilon}^{m}-g_{+}\right]_{+}\right\|_{L^{2}}^{2}+\delta\left\|\nabla\left[s_{\varepsilon}^{m}-g_{+}\right]_{+}\right\|_{L^{p}}^{p}\right]+k_{2}\left\|\left[s_{\varepsilon}^{m}-g_{+}\right]_{+} \nabla \varphi_{\varepsilon}^{m-1}\right\|_{L^{2}}^{2} \\
+\left(\widetilde{W}_{+}^{\prime}\left(s_{\varepsilon}^{m}\right)-\widetilde{W}_{+}^{\prime}\left(g_{+}\right),\left[s_{\varepsilon}^{m}-g_{+}\right]_{+}\right) \\
=\left(\frac{1}{\tau_{m}}\left[s_{\varepsilon}^{m-1}-g_{+}\right]-k_{2}\left|\nabla \varphi_{\varepsilon}^{m-1}\right|^{2} g_{+},\left[s_{\varepsilon}^{m}-g_{+}\right]_{+}\right)+\left(\left[\widetilde{W}_{-}^{\prime}\left(s_{\varepsilon}^{m-1}\right)-\widetilde{W}_{-}^{\prime}\left(g_{+}\right)\right]-W^{\prime}\left(g_{+}\right),\left[s_{\varepsilon}^{m}-g_{+}\right]_{+}\right) \leq 0 ;
\end{gathered}
$$

and hence that $s_{\varepsilon}^{m} \leq g_{+}$. Similarly, choosing $v=\left[s_{\varepsilon}^{m}-g_{-}\right]_{-} \in V_{0}\left(\varphi_{\varepsilon}^{m-1}\right)$ in (2.7a) yields that $s_{\varepsilon}^{m} \geq g_{-}$. Similarly, assuming that $\varphi_{\varepsilon}^{m-1} \in\left[\phi_{-}, \phi_{+}\right]$and choosing $\eta=\left[\varphi_{\varepsilon}^{m}-\phi_{+}\right]_{+},\left[\varphi_{\varepsilon}^{m}-\phi_{-}\right]_{-} \in H_{0}^{1}(\Omega)$ in (2.7b) yields that $\varphi_{\varepsilon}^{m} \in\left[\phi_{-}, \phi_{+}\right]$. As $\left\{s_{\varepsilon}^{0}, \varphi_{\varepsilon}^{0}\right\}=\{g, \phi\}$, it follows by induction for $m=1,2, \cdots M$ that

$$
s_{\varepsilon}^{m}(x) \in\left[g_{-}, g_{+}\right] \quad \text { and } \quad \varphi_{\varepsilon}^{m}(x) \in\left[\phi_{-}, \phi_{+}\right] \quad \text { for a.e. } x \in \Omega \text {. }
$$

Moreover, on choosing $v=d_{t} s_{\varepsilon}^{m}$ in (2.7a) and $\eta=\frac{k_{2}}{k_{3}} d_{t} \varphi_{\varepsilon}^{m}$ in (2.7b), and adding yields that

$$
\begin{array}{r}
\left.\left\|d_{t} s_{\varepsilon}^{m}\right\|_{L^{2}}^{2}+\frac{k_{1}}{2}\left[d_{t}\left\|\nabla s_{\varepsilon}^{m}\right\|_{L^{2}}^{2}+\tau_{m}\left\|d_{t} \nabla s_{\varepsilon}^{m}\right\|_{L^{2}}^{2}\right]+k_{1} \delta\left(\left|\nabla s_{\varepsilon}^{m}\right|^{p-2} \nabla s_{\varepsilon}^{m}, \nabla\left(d_{t} s_{\varepsilon}^{m}\right)\right)+\frac{k_{2}}{k_{3}} \|\left(s_{\varepsilon}^{m}\right)^{2}+\varepsilon^{2}\right)^{\frac{1}{2}} d_{t} \varphi_{\varepsilon}^{m} \|_{L^{2}}^{2} \\
+\frac{k_{2}}{2}\left[\left(\left|\nabla \varphi_{\varepsilon}^{m-1}\right|^{2}, d_{t}\left(s_{\varepsilon}^{m}\right)^{2}+\tau_{m}\left(d_{t} s_{\varepsilon}^{m}\right)^{2}\right)+\left(\left(s_{\varepsilon}^{m}\right)^{2}+\varepsilon^{2}, d_{t}\left|\nabla \varphi_{\varepsilon}^{m}\right|^{2}+\tau_{m}\left|d_{t} \nabla \varphi_{\varepsilon}^{m}\right|^{2}\right)\right]+\left(\widetilde{W}_{+}^{\prime}\left(s_{\varepsilon}^{m}\right), d_{t} s_{\varepsilon}^{m}\right) \\
=\left(\widetilde{W}_{-}^{\prime}\left(s_{\varepsilon}^{m-1}\right), d_{t} s_{\varepsilon}^{m}\right) .
\end{array}
$$

The convexity of $|\nabla \cdot|^{p}$ for $p>1$ and $\widetilde{W}_{ \pm}$imply that

$$
\begin{aligned}
& \tau_{m}\left|\nabla v^{m}\right|^{p-2} \nabla v^{m} \cdot \nabla\left(d_{t} v^{m}\right) \geq \frac{1}{p}\left[\left|\nabla v^{m}\right|^{p}-\left|\nabla v^{m-1}\right|^{p}\right] \\
& \widetilde{W}_{ \pm}^{\prime}\left(v^{m}\right) d_{t} v^{m} \geq d_{t} \widetilde{W}_{ \pm}\left(v^{m}\right) \geq \widetilde{W}_{ \pm}^{\prime}\left(v^{m-1}\right) d_{t} v^{m}
\end{aligned}
$$

Also, a direct calculation yields that

$$
\left|\nabla \eta^{m-1}\right|^{2} d_{t}\left(v^{m}\right)^{2}+\left[\left(v^{m}\right)^{2}+\varepsilon^{2}\right] d_{t}\left|\nabla \eta^{m}\right|^{2}=d_{t}\left(\left[\left(v^{m}\right)^{2}+\varepsilon^{2}\right]\left|\nabla \eta^{m}\right|^{2}\right) .
$$


Applying the operator $\sum_{m=1}^{\ell} \tau_{m}$, to (2.12) and noting (2.13a,b) and (2.14), yields for $\ell=1,2, \cdots M$ that

$$
\begin{aligned}
& \frac{1}{2}\left\{k_{1}\left\|\nabla s_{\varepsilon}^{\ell}\right\|_{L^{2}}^{2}+k_{2}\left\|\left(\left(s_{\varepsilon}^{\ell}\right)^{2}+\varepsilon^{2}\right)^{\frac{1}{2}} \nabla \varphi_{\varepsilon}^{\ell}\right\|_{L^{2}}^{2}\right\}+\frac{k_{1} \delta}{p}\left\|\nabla s_{\varepsilon}^{\ell}\right\|_{L^{p}}^{p}+\int_{\Omega} \widetilde{W}\left(s_{\varepsilon}^{\ell}\right) \mathrm{d} x \\
& +\sum_{m=1}^{\ell} \tau_{m}\left\{\left\|d_{t} s_{\varepsilon}^{m}\right\|_{L^{2}}^{2}+\frac{k_{2}}{k_{3}}\left\|\left(\left(s_{\varepsilon}^{m}\right)^{2}+\varepsilon^{2}\right)^{\frac{1}{2}} d_{t} \varphi_{\varepsilon}^{m}\right\|_{L^{2}}^{2}+\frac{k_{1}}{2} \tau_{m}\left\|d_{t} \nabla s_{\varepsilon}^{m}\right\|_{L^{2}}^{2}\right. \\
& \left.\quad+\frac{k_{2}}{2} \tau_{m}\left[\left\|\left(d_{t} s_{\varepsilon}^{m}\right) \nabla \varphi_{\varepsilon}^{m-1}\right\|_{L^{2}}^{2}+\left\|\left(\left(s_{\varepsilon}^{m}\right)^{2}+\varepsilon^{2}\right)^{\frac{1}{2}} d_{t} \nabla \varphi_{\varepsilon}^{m}\right\|_{L^{2}}^{2}\right]\right\} \\
& \leq \frac{1}{2}\left\{k_{1}\|\nabla g\|_{L^{2}}^{2}+k_{2}\left\|\left(g^{2}+\varepsilon^{2}\right)^{\frac{1}{2}} \nabla \phi\right\|_{L^{2}}^{2}\right\}+\frac{k_{1} \delta}{p}\|\nabla g\|_{L^{p}}^{p}+\int_{\Omega} \widetilde{W}(g) \mathrm{d} x \leq C,
\end{aligned}
$$

where $C$ is independent of $\tau$ and $\varepsilon$.

Let

$$
\begin{array}{llll}
s_{\varepsilon, \tau}(\cdot, t):=\frac{t-t_{m-1}}{\tau_{m}} s_{\varepsilon}^{m}(\cdot)+\frac{t_{m}-t}{\tau_{m}} s_{\varepsilon}^{m-1}(\cdot) & t \in\left[t_{m-1}, t_{m}\right] & m=1,2, \cdots M, \\
s_{\varepsilon, \tau}^{+}(\cdot, t):=s_{\varepsilon}^{m}(\cdot), \quad s_{\varepsilon, \tau}^{-}(\cdot, t):=s_{\varepsilon}^{m-1}(\cdot) & t \in\left(t_{m-1}, t_{m}\right] & m=1,2, \cdots M .
\end{array}
$$

Using the above notation, and introducing analogous notation for $\varphi_{\varepsilon}$, and noting $(2.11)$ and $(2.6),\left(\mathrm{P}_{\delta, \varepsilon}^{\tau}\right)$ can be restated as: Find $\left\{s_{\varepsilon, \tau}, \varphi_{\varepsilon, \tau}\right\} \in L^{\infty}\left(0, T ; W_{g}^{1, p^{\star}}(\Omega)\right) \times L^{\infty}\left(0, T ; H_{\phi}^{1}(\Omega)\right)$ such that $s_{\varepsilon, \tau}^{+} \nabla \varphi_{\varepsilon, \tau}^{-} \in L^{\infty}\left(0, T ;\left[L^{2}(\Omega)\right]^{d}\right)$, $s_{\varepsilon, \tau}(\cdot, 0)=g(\cdot), \varphi_{\varepsilon, \tau}(\cdot, 0)=\phi(\cdot)$ and

$$
\begin{array}{cc}
\int_{\Omega_{T}}\left\{\partial_{t} s_{\varepsilon, \tau} v+k_{1}\left[1+\delta\left|\nabla s_{\varepsilon, \tau}^{+}\right|^{p-2}\right] \nabla s_{\varepsilon, \tau}^{+} \cdot \nabla v+k_{2}\left|\nabla \varphi_{\varepsilon, \tau}^{-}\right|^{2} s_{\varepsilon, \tau}^{+} v+\left[W_{+}^{\prime}\left(s_{\varepsilon, \tau}^{+}\right)-W_{-}^{\prime}\left(s_{\varepsilon, \tau}^{-}\right)\right] v\right\} \mathrm{d} x \mathrm{~d} t=0 \\
\int_{\Omega_{T}}\left[\left(s_{\varepsilon, \tau}^{+}\right)^{2}+\varepsilon^{2}\right]\left\{\partial_{t} \varphi_{\varepsilon, \tau} \eta+k_{3} \nabla \varphi_{\varepsilon, \tau}^{+} . \nabla \eta\right\} \mathrm{d} x \mathrm{~d} t=0 & \forall v \in L^{2}\left(0, T ; W_{0}^{1, p^{\star}}(\Omega) \cap L^{\infty}(\Omega)\right),
\end{array}
$$

Moreover, the bounds (2.15) and a Poincaré inequality immediately yield that

$$
\begin{aligned}
& s_{\varepsilon, \tau}^{( \pm)} \in L^{\infty}\left((0, T) ; W^{1, p^{\star}}(\Omega)\right), \quad \tau^{-\frac{1}{2}}\left(s_{\varepsilon, \tau}^{+}-s_{\varepsilon, \tau}^{-}\right) \in L^{2}\left(0, T ; H_{0}^{1}(\Omega)\right), \quad s_{\varepsilon, \tau} \in H^{1}\left((0, T) ; L^{2}(\Omega)\right), \\
& \left(\left(s_{\varepsilon, \tau}^{ \pm}\right)^{2}+\varepsilon^{2}\right)^{\frac{1}{2}} \nabla \varphi_{\varepsilon, \tau}^{ \pm} \in L^{\infty}\left((0, T) ;\left[L^{2}(\Omega)\right]^{d}\right), \quad \tau^{-\frac{1}{2}}\left(\left(s_{\varepsilon, \tau}^{+}\right)^{2}+\varepsilon^{2}\right)^{\frac{1}{2}} \nabla\left(\varphi_{\varepsilon, \tau}^{+}-\varphi_{\varepsilon, \tau}^{-}\right) \in L^{2}\left(0, T ;\left[L^{2}(\Omega)\right]^{d}\right), \\
& \tau^{-\frac{1}{2}}\left|\nabla \varphi_{\varepsilon, \tau}^{-}\right|\left(s_{\varepsilon, \tau}^{+}-s_{\varepsilon, \tau}^{-}\right) \in L^{2}\left(\Omega_{T}\right), \quad\left(\left(s_{\varepsilon, \tau}^{+}\right)^{2}+\varepsilon^{2}\right)^{\frac{1}{2}} \partial_{t} \varphi_{\varepsilon, \tau} \in L^{2}\left(\Omega_{T}\right) ;
\end{aligned}
$$

and their respective norms are independent of $\tau$ and $\varepsilon$. In addition (2.11) implies that

$$
s_{\varepsilon, \tau}^{( \pm)}(x, t) \in\left[g_{-}, g_{+}\right], \quad \varphi_{\varepsilon, \tau}^{( \pm)}(x, t) \in\left[\phi_{-}, \phi_{+}\right] \quad \text { for a.e. }(x, t) \in \Omega_{T} .
$$

The uniform estimates (2.18) and (2.19), a generalised Lebesgue dominated convergence theorem and the compact embedding $W^{1, p}(\Omega) \stackrel{c}{\hookrightarrow} C(\bar{\Omega})$ for $p>d$ immediately imply that there exist $\left\{s_{\varepsilon}, \varphi_{\varepsilon}\right\}$ satisfying (i)-(iii) of Definition 2.1 and the following convergence results for a subsequence of $\left\{s_{\varepsilon, \tau}^{( \pm)}, \varphi_{\varepsilon, \tau}^{( \pm)}\right\}_{\tau>0}$ as $\tau \rightarrow 0$ :

$$
\begin{aligned}
& \partial_{t} s_{\varepsilon, \tau} \longrightarrow \partial_{t} s_{\varepsilon} \quad \text { weakly in } L^{2}\left(\Omega_{T}\right) \text {; } \\
& s_{\varepsilon, \tau}^{( \pm)} \longrightarrow s_{\varepsilon} \quad \text { weak* in } L^{\infty}\left(0, T ; W^{1, p^{*}}(\Omega)\right), \quad \text { strongly in } L^{q_{1}}\left(\Omega_{T}\right) \text {; } \\
& \partial_{t} \varphi_{\varepsilon, \tau} \longrightarrow \partial_{t} \varphi_{\varepsilon} \quad \text { weakly in } L^{2}\left(\Omega_{T}\right) \text {; } \\
& \varphi_{\varepsilon, \tau}^{( \pm)} \longrightarrow \varphi_{\varepsilon} \quad \text { weak* in } L^{\infty}\left(0, T ; H^{1}(\Omega)\right), \quad \text { strongly in } L^{q_{2}}\left(\Omega_{T}\right) ;
\end{aligned}
$$


where $q_{i} \in[1, \infty)$, but if either (a) $d=1$ or (b) $\delta>0$ then $q_{1} \in[1, \infty]$; and hence

$$
\begin{aligned}
\left(\left(s_{\varepsilon, \tau}^{+}\right)^{2}+\varepsilon^{2}\right) \partial_{t} \varphi_{\varepsilon, \tau} \longrightarrow\left(s_{\varepsilon}^{2}+\varepsilon^{2}\right) \partial_{t} \varphi_{\varepsilon} & \text { weakly in } L^{2}\left(\Omega_{T}\right) ; \\
\left(\left(s_{\varepsilon, \tau}^{+}\right)^{2}+\varepsilon^{2}\right) \nabla \varphi_{\varepsilon, \tau}^{+} \longrightarrow\left(s_{\varepsilon}^{2}+\varepsilon^{2}\right) \nabla \varphi_{\varepsilon} & \text { weak* in } L^{\infty}\left(0, T ;\left[L^{2}(\Omega)\right]^{d}\right) ; \\
s_{\varepsilon, \tau}^{+} \nabla \varphi_{\varepsilon, \tau}^{ \pm} \longrightarrow s_{\varepsilon} \nabla \varphi_{\varepsilon} & \text { weak* in } L^{\infty}\left(0, T ;\left[L^{2}(\Omega)\right]^{d}\right) ;
\end{aligned}
$$

where for brevity we adopt the same notation for the subsequence.

It follows from (2.21), that we can pass to the limit $\tau \rightarrow 0$ for the subsequence of (2.17b) to obtain (2.4b). In order to pass to the corresponding limit in (2.17a), we require some stronger convergence for the third term.

From (2.17b) with $\eta=\varphi_{\varepsilon, \tau}^{+}-\phi,(2.20),(2.21)$ and (2.4b) with $\eta=\varphi_{\varepsilon}-\phi$ we obtain that

$$
\begin{aligned}
\limsup _{\tau \rightarrow 0} \int_{\Omega_{T}}\left(\left(s_{\varepsilon, \tau}^{+}\right)^{2}+\varepsilon^{2}\right)\left|\nabla \varphi_{\varepsilon, \tau}^{+}\right|^{2} \mathrm{~d} x \mathrm{~d} t & =\limsup _{\tau \rightarrow 0} \int_{\Omega_{T}}\left(\left(s_{\varepsilon, \tau}^{+}\right)^{2}+\varepsilon^{2}\right)\left[\nabla \varphi_{\varepsilon, \tau}^{+} \cdot \nabla \phi-k_{3}^{-1} \partial_{t} \varphi_{\varepsilon, \tau}\left(\varphi_{\varepsilon, \tau}^{+}-\phi\right)\right] \mathrm{d} x \mathrm{~d} t \\
& =\int_{\Omega_{T}}\left(s_{\varepsilon}^{2}+\varepsilon^{2}\right)\left[\nabla \varphi_{\varepsilon} \cdot \nabla \phi-k_{3}^{-1} \partial_{t} \varphi_{\varepsilon}\left(\varphi_{\varepsilon}-\phi\right)\right] \mathrm{d} x \mathrm{~d} t \\
& =\int_{\Omega_{T}}\left(s_{\varepsilon}^{2}+\varepsilon^{2}\right)\left|\nabla \varphi_{\varepsilon}\right|^{2} \mathrm{~d} x \mathrm{~d} t
\end{aligned}
$$

It follows from (2.20), (2.21) and (2.22) that

$$
\begin{aligned}
& \limsup _{\tau \rightarrow 0} \int_{\Omega_{T}}\left|\left(\left(s_{\varepsilon, \tau}^{+}\right)^{2}+\varepsilon^{2}\right)^{\frac{1}{2}} \nabla \varphi_{\varepsilon, \tau}^{+}-\left(s_{\varepsilon}^{2}+\varepsilon^{2}\right)^{\frac{1}{2}} \nabla \varphi_{\varepsilon}\right|^{2} \mathrm{~d} x \mathrm{~d} t \\
& \quad=\limsup _{\tau \rightarrow 0} \int_{\Omega_{T}}\left\{\left(\left(s_{\varepsilon, \tau}^{+}\right)^{2}+\varepsilon^{2}\right)\left|\nabla \varphi_{\varepsilon, \tau}^{+}\right|^{2}-2\left[\left(s_{\varepsilon}^{2}+\varepsilon^{2}\right)\left(\left(s_{\varepsilon, \tau}^{+}\right)^{2}+\varepsilon^{2}\right)\right]^{\frac{1}{2}} \nabla \varphi_{\varepsilon, \tau}^{+} \cdot \nabla \varphi_{\varepsilon}+\left(s_{\varepsilon}^{2}+\varepsilon^{2}\right)\left|\nabla \varphi_{\varepsilon}\right|^{2}\right\} \mathrm{d} x \mathrm{~d} t \\
& \quad=\limsup _{\tau \rightarrow 0} \int_{\Omega_{T}}\left\{\left(\left(s_{\varepsilon, \tau}^{+}\right)^{2}+\varepsilon^{2}\right)\left|\nabla \varphi_{\varepsilon, \tau}^{+}\right|^{2}-\left(s_{\varepsilon}^{2}+\varepsilon^{2}\right)\left|\nabla \varphi_{\varepsilon}\right|^{2}\right\} \mathrm{d} x \mathrm{~d} t=0 .
\end{aligned}
$$

Combining (2.23) and (2.18) we obtain that

$$
\left(\left(s_{\varepsilon, \tau}^{+}\right)^{2}+\varepsilon^{2}\right)^{\frac{1}{2}} \nabla \varphi_{\varepsilon, \tau}^{ \pm} \longrightarrow\left(s_{\varepsilon}^{2}+\varepsilon^{2}\right)^{\frac{1}{2}} \nabla \varphi_{\varepsilon} \quad \text { strongly in } L^{2}\left(0, T ;\left[L^{2}(\Omega)\right]^{d}\right) \text { as } \tau \rightarrow 0 .
$$

Therefore we obtain from (2.24) and (2.20) on extracting a further subsequence and applying a generalised Lebesgue dominated convergence theorem that

$$
s_{\varepsilon, \tau}^{+} \nabla \varphi_{\varepsilon, \tau}^{ \pm} \longrightarrow s_{\varepsilon} \nabla \varphi_{\varepsilon} \quad \text { strongly in } L^{2}\left(0, T ;\left[L^{2}(\Omega)\right]^{d}\right) \text { as } \tau \rightarrow 0 .
$$

If $\delta=0$, it follows from $(2.20),(2.25),(2.19)$ that we can pass to the limit $\tau \rightarrow 0$ for a subsequence of (2.17a) to obtain $(2.4 \mathrm{a})$ with $\delta=0$. In order to achieve the corresponding limit in the case $\delta>0$, we have to exploit "the decisive monotonicity trick", see ([8], p. 474). It follows from the monotonicity of $|a|^{p-2} a, a \in \mathbb{R}^{d}$, and (2.17a) that for all $\lambda \in \mathbb{R}$ and for all $v \in L^{2}\left((0, T) ; W_{0}^{1, p^{\star}}(\Omega) \cap L^{\infty}(\Omega)\right)$

$$
\begin{aligned}
0 \leq k_{1} \int_{\Omega_{T}}\left\{\left[1+\delta\left|\nabla\left(s_{\varepsilon}+\lambda v\right)\right|^{p-2}\right] \nabla\left(s_{\varepsilon}+\lambda v\right)-\left[1+\delta\left|\nabla s_{\varepsilon, \tau}^{+}\right|^{p-2}\right] \nabla s_{\varepsilon, \tau}^{+}\right\} \cdot \nabla\left[s_{\varepsilon}+\lambda v-s_{\varepsilon, \tau}^{+}\right] \mathrm{d} x \mathrm{~d} t \\
=k_{1} \int_{\Omega_{T}}\left[1+\delta\left|\nabla\left(s_{\varepsilon}+\lambda v\right)\right|^{p-2}\right] \nabla\left(s_{\varepsilon}+\lambda v\right) \cdot \nabla\left[s_{\varepsilon}+\lambda v-s_{\varepsilon, \tau}^{+}\right] \mathrm{d} x \mathrm{~d} t \\
\quad+\int_{\Omega_{T}}\left[\partial_{t} s_{\varepsilon, \tau}+k_{2}\left|\nabla \varphi_{\varepsilon, \tau}^{-}\right|^{2} s_{\varepsilon, \tau}^{+}+W_{+}^{\prime}\left(s_{\varepsilon, \tau}^{+}\right)-W_{-}^{\prime}\left(s_{\varepsilon, \tau}^{-}\right)\right]\left(s_{\varepsilon}+\lambda v-s_{\varepsilon, \tau}^{+}\right) \mathrm{d} x \mathrm{~d} t .
\end{aligned}
$$


On noting (2.20), (2.25) and (2.19), we now pass to the limit $\tau \rightarrow 0$ in a subsequence of (2.26) yielding for all $\lambda \in \mathbb{R}$ and for all $v \in L^{2}\left((0, T) ; W_{0}^{1, p^{\star}}(\Omega) \cap L^{\infty}(\Omega)\right)$ that

$$
0 \leq \lambda \int_{\Omega_{T}}\left\{k_{1}\left[1+\delta\left|\nabla\left(s_{\varepsilon}+\lambda v\right)\right|^{p-2}\right] \nabla\left(s_{\varepsilon}+\lambda v\right) \cdot \nabla v+\left[\partial_{t} s_{\varepsilon}+k_{2} s_{\varepsilon}\left|\nabla \varphi_{\varepsilon}\right|^{2}+W^{\prime}\left(s_{\varepsilon}\right)\right] v\right\} \mathrm{d} x \mathrm{~d} t .
$$

Considering the cases of $\lambda>0$ and $\lambda<0$ separately, we divide (2.27) by $\lambda$ and then pass to the limit $\lambda \rightarrow 0$ to obtain the desired result (2.4a). Therefore we have proved global existence of a weak solution to $\left(\mathrm{P}_{\delta, \varepsilon}\right)$ for fixed $\delta \geq 0, p>d$ and $\varepsilon \in(0,1]$, subject to $(2.1)$ and (2.2).

Finally, it follows from $(2.8 \mathrm{~b}),(2.15),(2.20),(2.21),(2.13 \mathrm{a}),(2.19)$ and $(2.6)$ that for a.a. $\tilde{t} \in(0, T)$

$$
\begin{aligned}
& E_{\varepsilon}\left(s_{\varepsilon}, \varphi_{\varepsilon}\right)(\tilde{t})+\int_{\Omega_{\tilde{t}}}\left\{\left(\partial_{t} s_{\varepsilon}\right)^{2}+\frac{k_{2}}{k_{3}}\left(s_{\varepsilon}^{2}+\varepsilon^{2}\right)\left(\partial_{t} \varphi_{\varepsilon}\right)^{2}\right\} \mathrm{d} x \mathrm{~d} t \\
& \quad \leq \liminf _{\tau \rightarrow 0}\left[E_{\varepsilon}\left(s_{\varepsilon, \tau}^{+}, \varphi_{\varepsilon, \tau}^{+}\right)(\tilde{t})+\int_{\Omega_{\tilde{t}}}\left\{\left(\partial_{t} s_{\varepsilon, \tau}\right)^{2}+\frac{k_{2}}{k_{3}}\left(\left(s_{\varepsilon, \tau}^{+}\right)^{2}+\varepsilon^{2}\right)\left(\partial_{t} \varphi_{\varepsilon, \tau}\right)^{2}\right\} \mathrm{d} x \mathrm{~d} t\right] \leq E_{\varepsilon}(g, \phi),
\end{aligned}
$$

and hence the desired dissipative energy law (2.8a).

In the theorem below, we show uniqueness of weak solutions to $\left(\mathrm{P}_{\delta, \varepsilon}\right)$ in the case $d=1$. However, for $d=2$ or 3 we require slightly stronger regularity.

Theorem 2.5. For any fixed $\delta \geq 0, p>d$ and $\varepsilon \in(0,1]$, the system $\left(P_{\delta, \varepsilon}\right)$ subject to $(2.1)$ and $(2.2)$ possesses at most one weak solution $\left\{s_{\varepsilon}, \varphi_{\varepsilon}\right\}$ in the function class $\left[L^{4}\left((0, T) ; W^{1,2 d}(\Omega)\right) \cap H^{1}\left((0, T) ; L^{d}(\Omega)\right)\right]^{2}$.

Proof. Suppose $\left\{s_{\varepsilon}^{(j)}, \varphi_{\varepsilon}^{(j)}\right\} \in\left[L^{4}\left((0, T) ; W^{1,2 d}(\Omega)\right) \cap H^{1}\left((0, T) ; L^{d}(\Omega)\right)\right]^{2}, j=1$ and 2 , are two weak solutions corresponding to the same initial and boundary data. Obviously, this not a constraint if $d=1$. Let $\bar{s}_{\varepsilon}:=$ $s_{\varepsilon}^{(1)}-s_{\varepsilon}^{(2)}$ and $\bar{\varphi}_{\varepsilon}:=\varphi_{\varepsilon}^{(1)}-\varphi_{\varepsilon}^{(2)}$. It suffices to show that $\bar{s}_{\varepsilon}=0$ and $\bar{\varphi}_{\varepsilon}=0$ a.e. in $\Omega_{T}$.

It is easy to check that $\left\{\bar{s}_{\varepsilon}, \bar{\varphi}_{\varepsilon}\right\}$ satisfies $\bar{s}_{\varepsilon}(x, 0)=\bar{\varphi}_{\varepsilon}(x, 0)=0$ for a.e. $x \in \Omega, \bar{s}_{\varepsilon}(x, t)=\bar{\varphi}_{\varepsilon}(x, t)=0$ for a.e. $(x, t) \in \partial \Omega_{T}$, and the following "error" equations for all $\tilde{t} \in(0, T)$

$$
\begin{gathered}
\int_{\Omega_{\tilde{t}}}\left\{\partial_{t} \bar{s}_{\varepsilon} v+k_{1}\left[\nabla \bar{s}_{\varepsilon}+\delta\left(\left|\nabla s_{\varepsilon}^{(1)}\right|^{p-2} \nabla s_{\varepsilon}^{(1)}-\left|\nabla s_{\varepsilon}^{(2)}\right|^{p-2} \nabla s_{\varepsilon}^{(2)}\right)\right] \cdot \nabla v+k_{2}\left|\nabla \varphi_{\varepsilon}^{(2)}\right|^{2} \bar{s}_{\varepsilon} v\right. \\
\left.+k_{2}\left[\left|\nabla \varphi_{\varepsilon}^{(1)}\right|^{2}-\left|\nabla \varphi_{\varepsilon}^{(2)}\right|^{2}\right] s_{\varepsilon}^{(1)} v+W^{\prime \prime}(\xi) \bar{s}_{\varepsilon} v\right\} \mathrm{d} x \mathrm{~d} t=0 \quad \forall v \in L^{2}\left(0, \tilde{t}, W_{0}^{1, p^{\star}}(\Omega) \cap L^{\infty}(\Omega)\right), \\
\int_{\Omega_{\tilde{t}}}\left\{\left[\left(s_{\varepsilon}^{(1)}\right)^{2}+\varepsilon^{2}\right] \partial_{t} \bar{\varphi}_{\varepsilon} \eta+\left[s_{\varepsilon}^{(1)}+s_{\varepsilon}^{(2)}\right] \bar{s}_{\varepsilon} \partial_{t} \varphi_{\varepsilon}^{(2)} \eta+k_{3}\left[\left(s_{\varepsilon}^{(1)}\right)^{2}+\varepsilon^{2}\right] \nabla \bar{\varphi}_{\varepsilon} \cdot \nabla \eta\right. \\
\left.+k_{3}\left[s_{\varepsilon}^{(1)}+s_{\varepsilon}^{(2)}\right] \bar{s}_{\varepsilon} \nabla \varphi_{\varepsilon}^{(2)} \cdot \nabla \eta\right\} \mathrm{d} x \mathrm{~d} t=0 \quad \forall \eta \in L^{2}\left(0, \tilde{t}, H_{0}^{1}(\Omega)\right)
\end{gathered}
$$

where $\xi(x, t)$ lies between $s_{\varepsilon}^{(1)}(x, t)$ and $s_{\varepsilon}^{(2)}(x, t)$. Choosing $v=\bar{s}_{\varepsilon}$ in (2.29a) and $\eta=\bar{\varphi}_{\varepsilon}$ in (2.29b), and noting the monotonicity of $|a|^{p-2} a, a \in \mathbb{R}^{d}$, yields that

$$
\begin{aligned}
& \frac{1}{2}\left\|\bar{s}_{\varepsilon}(\cdot, \tilde{t})\right\|_{L^{2}}^{2}+\int_{0}^{\tilde{t}}\left\{k_{1}\left\|\nabla \bar{s}_{\varepsilon}\right\|_{L^{2}}^{2}+k_{2}\left\|\bar{s}_{\varepsilon} \nabla \varphi_{\varepsilon}^{(2)}\right\|_{L^{2}}^{2}\right\} \mathrm{d} t \\
& \quad \leq-\int_{\Omega_{\tilde{t}}}\left\{k_{2}\left[\left|\nabla \varphi_{\varepsilon}^{(1)}\right|^{2}-\left|\nabla \varphi_{\varepsilon}^{(2)}\right|^{2}\right] s_{\varepsilon}^{(1)} \bar{s}_{\varepsilon}+W^{\prime \prime}(\xi)\left|\bar{s}_{\varepsilon}\right|^{2}\right\} \mathrm{d} x \mathrm{~d} t, \\
& \frac{1}{2}\left\|\left(\left(s_{\varepsilon}^{(1)}(\cdot, \tilde{t})\right)^{2}+\varepsilon^{2}\right)^{\frac{1}{2}} \bar{\varphi}_{\varepsilon}(\cdot, \tilde{t})\right\|_{L^{2}}^{2}+k_{3} \int_{0}^{\tilde{t}}\left\|\left(\left(s_{\varepsilon}^{(1)}\right)^{2}+\varepsilon^{2}\right)^{\frac{1}{2}} \nabla \bar{\varphi}_{\varepsilon}\right\|_{L^{2}}^{2} \mathrm{~d} t \\
& =-\int_{\Omega_{\tilde{t}}}\left\{\left[\left(s_{\varepsilon}^{(1)}\right)^{2}-\left(s_{\varepsilon}^{(2)}\right)^{2}\right]\left(\left(\partial_{t} \varphi_{\varepsilon}^{(2)}\right) \bar{\varphi}_{\varepsilon}+k_{3} \nabla \varphi_{\varepsilon}^{(2)} \cdot \nabla \bar{\varphi}_{\varepsilon}\right)-s_{\varepsilon}^{(1)}\left(\partial_{t} s_{\varepsilon}^{(1)}\right)\left|\bar{\varphi}_{\varepsilon}\right|^{2}\right\} \mathrm{d} x \mathrm{~d} t ;
\end{aligned}
$$


where we have applied the initial and boundary data. On noting (ii) of Definition 2.1 and that

$$
\|\eta\|_{L^{r}}^{2} \leq C\|\eta\|_{L^{2}}\|\nabla \eta\|_{L^{2}} \quad \forall \eta \in H_{0}^{1}(\Omega), \quad \text { where } r= \begin{cases}\infty & \text { if } d=1 \\ \frac{2 d}{d-1} & \text { if } d \geq 2\end{cases}
$$

the right hand sides of $(2.30 \mathrm{a}, \mathrm{b})$ can be bounded as follows

$$
\begin{aligned}
& \left|\int_{\Omega_{\tilde{t}}}\left\{k_{2}\left[\left|\nabla \varphi_{\varepsilon}^{(1)}\right|^{2}-\left|\nabla \varphi_{\varepsilon}^{(2)}\right|^{2}\right] s_{\varepsilon}^{(1)} \bar{s}_{\varepsilon}+W^{\prime \prime}(\xi)\left|\bar{s}_{\varepsilon}\right|^{2}\right\} \mathrm{d} x \mathrm{~d} t\right| \\
& \leq C \int_{0}^{\tilde{t}}\left\{k_{2}\left\|\nabla\left(\varphi_{\varepsilon}^{(1)}+\varphi_{\varepsilon}^{(2)}\right)\right\|_{L^{2 d}}\left\|s_{\varepsilon}^{(1)} \nabla \bar{\varphi}_{\varepsilon}\right\|_{L^{2}}\left[\left\|\bar{s}_{\varepsilon}\right\|_{L^{2}}\left\|\nabla \bar{s}_{\varepsilon}\right\|_{L^{2}}\right]^{\frac{1}{2}}+\left\|\bar{s}_{\varepsilon}\right\|_{L^{2}}^{2}\right\} \mathrm{d} t \\
& \leq \int_{0}^{\tilde{t}}\left\{\frac{k_{3}}{2}\left\|s_{\varepsilon}^{(1)} \nabla \bar{\varphi}_{\varepsilon}\right\|_{L^{2}}^{2}+\frac{k_{1}}{2}\left\|\nabla \bar{s}_{\varepsilon}\right\|_{L^{2}}^{2}+C\left[1+\left\|\nabla\left(\varphi_{\varepsilon}^{(1)}+\varphi_{\varepsilon}^{(2)}\right)\right\|_{L^{2 d}}^{4}\right]\left\|\bar{s}_{\varepsilon}\right\|_{L^{2}}^{2}\right\} \mathrm{d} t, \\
& \left|\int_{\Omega_{\tilde{t}}}\left\{\left[\left(s_{\varepsilon}^{(1)}\right)^{2}-\left(s_{\varepsilon}^{(2)}\right)^{2}\right]\left(\left(\partial_{t} \varphi_{\varepsilon}^{(2)}\right) \bar{\varphi}_{\varepsilon}+k_{3} \nabla \varphi_{\varepsilon}^{(2)} \cdot \nabla \bar{\varphi}_{\varepsilon}\right)-s_{\varepsilon}^{(1)} \partial_{t} s_{\varepsilon}^{(1)}\left|\bar{\varphi}_{\varepsilon}\right|^{2}\right\} \mathrm{d} x \mathrm{~d} t\right| \\
& \leq C \int_{0}^{\tilde{t}}\left\{\left\|\partial_{t} \varphi_{\varepsilon}^{(2)}\right\|_{L^{d}}\left[\left\|\bar{s}_{\varepsilon}\right\|_{L^{2}}\left\|\nabla \bar{s}_{\varepsilon}\right\|_{L^{2}}\left\|\bar{\varphi}_{\varepsilon}\right\|_{L^{2}}\left\|\nabla \bar{\varphi}_{\varepsilon}\right\|_{L^{2}}\right]^{\frac{1}{2}}\right. \\
& \left.+\left\|\nabla \varphi_{\varepsilon}^{(2)}\right\|_{L^{2 d}}\left\|\nabla \bar{\varphi}_{\varepsilon}\right\|_{L^{2}}\left[\left\|\bar{s}_{\varepsilon}\right\|_{L^{2}}\left\|\nabla \bar{s}_{\varepsilon}\right\|_{L^{2}}\right]^{\frac{1}{2}}+\left\|\partial_{t} s_{\varepsilon}^{(1)}\right\|_{L^{d}}\left\|\bar{\varphi}_{\varepsilon}\right\|_{L^{2}}\left\|\nabla \bar{\varphi}_{\varepsilon}\right\|_{L^{2}}\right\} \mathrm{d} t \\
& \leq \int_{0}^{\tilde{t}}\left\{\frac{\varepsilon^{2} k_{3}}{2}\left\|\nabla \bar{\varphi}_{\varepsilon}\right\|_{L^{2}}^{2}+\frac{k_{1}}{2}\left\|\nabla \bar{s}_{\varepsilon}\right\|_{L^{2}}^{2}+C\left(\varepsilon^{-1}\right)\left[\left\|\partial_{t} s_{\varepsilon}^{(1)}\right\|_{L^{d}}^{2}+\left\|\partial_{t} \varphi_{\varepsilon}^{(2)}\right\|_{L^{d}}^{2}\right]\left\|\bar{\varphi}_{\varepsilon}\right\|_{L^{2}}^{2}\right. \\
& \left.+C\left(\varepsilon^{-1}\right)\left[\left\|\partial_{t} \varphi_{\varepsilon}^{(2)}\right\|_{L^{d}}^{2}+\left\|\nabla \varphi_{\varepsilon}^{(2)}\right\|_{L^{2 d}}^{4}\right]\left\|\bar{s}_{\varepsilon}\right\|_{L^{2}}^{2}\right\} \mathrm{d} t
\end{aligned}
$$

where $C(a)$ denotes a generic positive constant which depends on the parameter $a$. Combining (2.30a,b) and $(2.32 \mathrm{a}, \mathrm{b})$, applying Gronwall's inequality and noting the assumed regularity yields the desired result.

Corollary 2.6. For any fixed $\delta \geq 0, p>d$ and $\varepsilon \in(0,1]$ and, the system $\left(P_{\delta, \varepsilon}\right)$ subject to $(2.1)$ and $(2.2)$ possesses at most one strong solution if $d=2$.

Proof. The result follows immediately from Theorem 2.5, Definition 2.3 and the embedding $L^{\infty}\left(0, T ; H^{1}(\Omega)\right) \cap$ $L^{2}\left(0, T ; H^{2}(\Omega)\right) \hookrightarrow L^{4}\left(0, T ; W^{1,4}(\Omega)\right)$ as $d=2$.

We now show that $\left(\mathrm{P}_{0, \varepsilon}\right)$ subject to $(2.1)$ and $(2.2)$ has a strong solution for sufficiently smooth data if $d=2$.

Theorem 2.7. In addition to the assumptions of Definition 2.1, we assume that $d=2, g, \phi \in H^{2}(\Omega)$, and that $\Omega$ is either a convex polygonal domain or $\partial \Omega \in C^{2}$. Then for any fixed $\varepsilon>0$, the system $\left(P_{0, \varepsilon}\right)$ subject to $(2.1)$ and (2.2) has a unique strong solution for all $T>0$.

Proof. Let $\left\{s_{\varepsilon}, \varphi_{\varepsilon}\right\}$ be a weak solution of $\left(\mathrm{P}_{0, \varepsilon}\right)$, whose existence was established by Theorem 2.4 , we want to show that $\left\{s_{\varepsilon}, \varphi_{\varepsilon}\right\}$ actually belong to $\left[L^{2}\left((0, T) ; H^{2}(\Omega)\right)\right]^{2}$. To this end, it suffices to derive a priori estimates for $s_{\varepsilon}$ and $\varphi_{\varepsilon}$ in the $L^{2}\left((0, T) ; H^{2}(\Omega)\right)$ norm. Obviously, one can make the argument below fully rigorous by deriving the desired bounds for the approximation $\left(\mathrm{P}_{0, \varepsilon}^{\tau}\right)$ and then passing to the limit $\tau \rightarrow 0$. Firstly, one can rewrite $(2.4 \mathrm{~b})$ as

$$
\int_{\Omega_{T}}\left\{\left[\partial_{t} \varphi_{\varepsilon}-2 k_{3} s_{\varepsilon}\left(s_{\varepsilon}^{2}+\varepsilon^{2}\right)^{-1} \nabla s_{\varepsilon} \cdot \nabla \varphi_{\varepsilon}\right] \eta+k_{3} \nabla \varphi_{\varepsilon} \cdot \nabla \eta\right\} \mathrm{d} x \mathrm{~d} t=0 \quad \forall \eta \in L^{2}\left((0, T) ; H_{0}^{1}(\Omega) \cap L^{\infty}(\Omega)\right) .
$$


Using Caldererón-Zygmund and Gagliardo-Nirenberg inequalities (cf. [1]) we obtain from (2.4a) and (2.33), on noting the uniform bounds in (i) of Definition 2.1 and the assumptions on $g, \phi$ and $\Omega$, that

$$
\begin{aligned}
\int_{0}^{T}\left\|s_{\varepsilon}\right\|_{H^{2}}^{2} \mathrm{~d} t \leq C \int_{0}^{T}(1 & \left.+\left\|\partial_{t} s_{\varepsilon}\right\|_{L^{2}}^{2}+\left\|\nabla \varphi_{\varepsilon}\right\|_{L^{4}}^{4}\right) \mathrm{d} t \leq C(T)+C \int_{0}^{T}\left\|\nabla \varphi_{\varepsilon}\right\|_{L^{4}}^{4} \mathrm{~d} t \\
\int_{0}^{T}\left\|\varphi_{\varepsilon}\right\|_{H^{2}}^{2} \mathrm{~d} t & \leq C \int_{0}^{T}\left(1+\left\|\partial_{t} \varphi_{\varepsilon}\right\|_{L^{2}}^{2}+\varepsilon^{-2}\left\|\nabla s_{\varepsilon}\right\|_{L^{4}}^{2}\left\|\nabla \varphi_{\varepsilon}\right\|_{L^{4}}^{2}\right) \mathrm{d} t \\
& \leq C(T)+C\left(\varepsilon^{-1}\right) \int_{0}^{T}\left\|\nabla s_{\varepsilon}\right\|_{L^{2}}\left\|s_{\varepsilon}\right\|_{H^{2}}\left\|\nabla \varphi_{\varepsilon}\right\|_{L^{4}}^{2} \mathrm{~d} t \\
& \leq C(T)+C\left(\varepsilon^{-1}\right) \int_{0}^{T}\left\|\nabla \varphi_{\varepsilon}\right\|_{L^{4}}^{4} \mathrm{~d} t
\end{aligned}
$$

The desired result follows immediately from $(2.34 \mathrm{a}, \mathrm{b})$ if we can show that

$$
\int_{0}^{T}\left\|\nabla \varphi_{\varepsilon}\right\|_{L^{4}}^{4} \mathrm{~d} t \leq C\left(\varepsilon^{-1}, T\right)\left\{\int_{0}^{T}\left\|\varphi_{\varepsilon}\right\|_{H^{2}}^{2} \mathrm{~d} t\right\}^{\frac{q}{2}}
$$

for some $q<2$. Let $\mathcal{G}: L^{2}(\Omega) \rightarrow H_{0}^{1}(\Omega) \cap H^{2}(\Omega)$ be the inverse Laplacian satisfying

$$
(\nabla(\mathcal{G} f), \nabla \eta)=(f, \eta) \quad \forall \eta \in H_{0}^{1}(\Omega) .
$$

Then by Meyers theorem, [6], see also [3] for a similar application; we have from (2.4b) that there exists an $r(\varepsilon)>2$ such that for a.a. $t \in(0, T)$

$$
\begin{aligned}
\left\|\nabla \varphi_{\varepsilon}(\cdot, t)\right\|_{L^{r}} & \leq C\left(\varepsilon^{-1}\right)\left[1+\left\|\left(\nabla \mathcal{G}\left[\left(s_{\varepsilon}^{2}+\varepsilon^{2}\right) \partial_{t} \varphi_{\varepsilon}\right]\right)(\cdot, t)\right\|_{L^{r}}\right] \\
& \leq C\left(\varepsilon^{-1}\right)\left[1+\left\|\left(\nabla \mathcal{G}\left[\left(s_{\varepsilon}^{2}+\varepsilon^{2}\right) \partial_{t} \varphi_{\varepsilon}\right]\right)(\cdot, t)\right\|_{L^{2}}^{\frac{2}{r}}\left\|\left(\mathcal{G}\left[\left(s_{\varepsilon}^{2}+\varepsilon^{2}\right) \partial_{t} \varphi_{\varepsilon}\right]\right)(\cdot, t)\right\|_{H^{2}}^{\frac{r-2}{r}}\right],
\end{aligned}
$$

where we have applied a Gagliardo-Nirenberg inequality to obtain the last bound. From (2.4b), (2.36), (2.8a,b) and (ii) of Definition 2.1 it follows that

$$
\left\|\left(\nabla \mathcal{G}\left[\left(s_{\varepsilon}^{2}+\varepsilon^{2}\right) \partial_{t} \varphi_{\varepsilon}\right]\right)(\cdot, t)\right\|_{L^{2}} \leq\left\|\left[\left(s_{\varepsilon}^{2}+\varepsilon^{2}\right) \nabla \varphi_{\varepsilon}\right](\cdot, t)\right\|_{L^{2}} \leq C .
$$

Integrating (2.37) in time, applying a Calderón-Zygmund inequality, and noting (2.38) and (i) of Definition 2.1, yields that

$$
\int_{0}^{T}\left\|\nabla \varphi_{\varepsilon}\right\|_{L^{r}}^{\frac{2 r}{r-2}} \mathrm{~d} t \leq C\left(\varepsilon^{-1}\right)\left[T+\int_{0}^{T}\left\|\left(s_{\varepsilon}^{2}+\varepsilon^{2}\right) \partial_{t} \varphi_{\varepsilon}\right\|_{L^{2}}^{2} \mathrm{~d} t\right] \leq C\left(\varepsilon^{-1}, T\right) .
$$

We assume that $r \in(2,4)$, otherwise the desired $L^{2}\left(0, T ; H^{2}(\Omega)\right)$ bounds follow immediately from $(2.34 \mathrm{a}, \mathrm{b})$ and (2.39). From a Gagliardo-Nirenberg inequality and (2.39) we obtain that

$$
\begin{aligned}
\int_{0}^{T}\left\|\nabla \varphi_{\varepsilon}\right\|_{L^{4}}^{4} \mathrm{~d} t & \leq \int_{0}^{T}\left\|\nabla \varphi_{\varepsilon}\right\|_{L^{r}}^{r}\left\|\varphi_{\varepsilon}\right\|_{H^{2}}^{4-r} \mathrm{~d} t \leq\left\{\int_{0}^{T}\left\|\nabla \varphi_{\varepsilon}\right\|_{L^{r}}^{\frac{2 r}{r-2}} \mathrm{~d} t\right\}^{\frac{r-2}{2}}\left\{\int_{0}^{T}\left\|\varphi_{\varepsilon}\right\|_{H^{2}}^{2} \mathrm{~d} t\right\}^{\frac{4-r}{2}} \\
& \leq C\left(\varepsilon^{-1}, T\right)\left\{\int_{0}^{T}\left\|\varphi_{\varepsilon}\right\|_{H^{2}}^{2} \mathrm{~d} t\right\}^{\frac{4-r}{2}}
\end{aligned}
$$

and hence (2.35) with $q=4-r<2$. Therefore the proof is complete. 


\subsection{Passing to the limit as $\varepsilon \rightarrow 0$}

In this subsection we will establish the existence of weak solutions to the system $\left(\mathrm{P}_{\delta}\right)$, with either (a) $\delta=0$ if $d=1$ or (b) $\delta>0$ and $p>d$ if $d \geq 1$, subject to (2.1) and (2.2) by passing to the limit as $\varepsilon \rightarrow 0$ in $\left(\mathrm{P}_{\delta, \varepsilon}\right)$. We present a detailed proof here, since there are a number gaps in the proof given in [2] for $\delta=0$. As we have stated previously, we are unable to fill all these gaps for $\left(\mathrm{P}_{0}\right)$. Hence the introduction of the $p$-Laplacian term in $\left(\mathrm{P}_{\delta}\right)$ for $d \geq 2$.

Our main idea, in order to fill these gaps, is to show strong convergence of a subsequence of $\left\{s_{\varepsilon} \nabla \varphi_{\varepsilon}\right\}$ to $s \nabla \varphi$ in $L^{2}\left(\Omega_{T}\right)$ by adapting techniques from [7]. This can be achieved for $\delta \geq 0$. However, one needs also to establish either the weak convergence of a subsequence of $\left\{s \nabla \varphi_{\varepsilon}\right\}$ to $s \nabla \varphi$ in $L^{2}\left(\Omega_{T}\right)$ or a related result. This unfortunately gives rise to the restriction $\delta>0$ and $p>d$ if $d \geq 2$.

We begin with the definition of a weak solution to $\left(\mathrm{P}_{\delta}\right)$, for given $\delta \geq 0$ and $p>d$, subject to (2.1) and (2.2).

Definition 2.8. Under the assumptions of Definition 2.1, a pair of functions $\{s, \varphi\}$ is said to be a weak solution to $\left(\mathrm{P}_{\delta}\right)$, with $\delta \geq 0$ and $p>d$, subject to $(2.1)$ and $(2.2)$ if $\{s, \varphi\}$ satisfies

(i) $s \in H^{1}\left((0, T) ; L^{2}(\Omega)\right) \cap L^{\infty}\left((0, T) ; W_{g}^{1, p^{\star}}(\Omega)\right)$ and $s \varphi \in H^{1}\left((0, T) ; L^{2}(\Omega)\right) \cap L^{\infty}\left((0, T) ; H^{1}(\Omega)\right)$;

(ii) $s(x, t) \in\left[g_{-}, g_{+}\right]$and $\varphi(x, t) \in\left[\phi_{-}, \phi_{+}\right]$for a.e. $(x, t) \in \Omega_{T}$;

(iii) $(2.1)\left(\right.$ a) in $Y_{1}$, where $W^{1, p^{\star}}(\Omega) \stackrel{c}{\hookrightarrow} Y_{1}$, and $(s \varphi)(\cdot, 0)=(g \phi)(\cdot)$ in $Y_{2}$, where $H^{1}(\Omega) \stackrel{c}{\hookrightarrow} Y_{2}$, e.g. $Y_{1}=Y_{2}=$ $L^{2}(\Omega)$

(iv) $s \varphi=g \phi$ in $L^{\infty}\left(0, T ; H^{\frac{1}{2}}(\partial \Omega)\right)$;

(v) the following identities:

$$
\begin{array}{cc}
\int_{\Omega_{T}}\left\{\partial_{t}\left(s^{2}\right) v+k_{1}\left[1+\delta|\nabla s|^{p-2}\right]\left[\nabla\left(s^{2}\right) \cdot \nabla v+2|\nabla s|^{2} v\right]+2 s W^{\prime}(s) v\right\} \mathrm{d} x \mathrm{~d} t+2 k_{2} \int_{\{s \neq 0\}} s^{2}|\nabla \varphi|^{2} v \mathrm{~d} x \mathrm{~d} t=0 \\
\int_{\Omega_{T}}\left\{s^{2} \partial_{t} \varphi \eta+k_{3} s^{2} \nabla \varphi \cdot \nabla \eta\right\} \mathrm{d} x \mathrm{~d} t=0 & \forall v \in L^{2}\left((0, T) ; W_{0}^{1, p^{\star}}(\Omega) \cap L^{\infty}(\Omega)\right),
\end{array}
$$

Theorem 2.9. The degenerate system $\left(P_{\delta}\right)$, with either $(a) \delta=0$ if $d=1$ or $(b) \delta>0$ and $p>d$ if $d \geq 1$, subject to (2.1) and (2.2) has a weak solution for all $T>0$. Moreover, the weak solution satisfies the following dissipative energy law for a.a. $\tilde{t} \in(0, T)$

$$
E(s, \varphi)(\tilde{t})+\int_{\Omega_{\tilde{t}}}\left\{\left(\partial_{t} s\right)^{2}+\frac{k_{2}}{k_{3}} s^{2}\left(\partial_{t} \varphi\right)^{2}\right\} \mathrm{d} x \mathrm{~d} t \leq E(g, \phi)
$$

where

$$
E(s, \varphi)(t):=\int_{\Omega}\left\{k_{1}\left[\frac{1}{2}|\nabla s(x, t)|^{2}+\frac{\delta}{p}|\nabla s(x, t)|^{p}\right]+W(s(x, t))\right\} \mathrm{d} x+\frac{k_{2}}{2} \int_{\{s(\cdot, t) \neq 0\}} s^{2}(x, t)|\nabla \varphi(x, t)|^{2} \mathrm{~d} x
$$

Proof. The proof is based on the uniform estimates (in $\varepsilon$ ) from the energy law (2.8a,b) for the regularized problem and compactness arguments. The main difficulty comes from passing to the limit in the nonlinear gradient term of (2.4a). Since the proof is long, we divide it into four steps.

Step 1 (extracting convergent subsequences). From (ii) of Definition 2.1, we have that

$$
s_{\varepsilon}(x, t) \in\left[g_{-}, g_{+}\right], \quad \varphi_{\varepsilon}(x, t) \in\left[\phi_{-}, \phi_{+}\right] \quad \text { for a.e. }(x, t) \in \Omega_{T} .
$$

From (2.8a,b) and (2.43), we immediately deduce that

$$
\begin{aligned}
& s_{\varepsilon} \in L^{\infty}\left((0, T) ; W^{1, p^{\star}}(\Omega)\right) \cap H^{1}\left((0, T) ; L^{2}(\Omega)\right), \quad s_{\varepsilon} \varphi_{\varepsilon} \in L^{\infty}\left((0, T) ; H^{1}(\Omega)\right) \cap H^{1}\left((0, T) ; L^{2}(\Omega)\right), \\
& \left(s_{\varepsilon}^{2}+\varepsilon^{2}\right)^{\frac{1}{2}} \nabla \varphi_{\varepsilon} \in L^{\infty}\left((0, T) ;\left[L^{2}(\Omega)\right]^{d}\right), \quad\left(s_{\varepsilon}^{2}+\varepsilon^{2}\right)^{\frac{1}{2}} \partial_{t} \varphi_{\varepsilon} \in L^{2}\left(\Omega_{T}\right),
\end{aligned}
$$


and their respective norms are independent of $\varepsilon$. Similarly to (2.20) and (2.21), the uniform estimates (2.43) and (2.44) immediately imply that there exist $\{s, \phi\}$ satisfying (i), (ii) and (iii) of Definition 2.8 and the following convergence results for a subsequence of $\left\{s_{\varepsilon}, \varphi_{\varepsilon}\right\}_{\varepsilon>0}$ as $\varepsilon \rightarrow 0$ :

$$
\begin{aligned}
\partial_{t} s_{\varepsilon} & \longrightarrow \partial_{t} s & & \text { weakly in } L^{2}\left(\Omega_{T}\right) ; \\
s_{\varepsilon} & \longrightarrow s & & \text { weak* in } L^{\infty}\left(0, T ; W^{1, p^{*}}(\Omega)\right), \text { strongly in } L^{q_{1}}\left(\Omega_{T}\right) ; \\
s_{\varepsilon} & \longrightarrow s, \quad \varphi_{\varepsilon} \longrightarrow \varphi & & \text { weak* in } L^{\infty}\left(\Omega_{T}\right) ; \\
\partial_{t}\left(s_{\varepsilon} \varphi_{\varepsilon}\right) & \longrightarrow \partial_{t}(s \varphi) & & \text { weakly in } L^{2}\left(\Omega_{T}\right) ; \\
s_{\varepsilon} \varphi_{\varepsilon} & \longrightarrow s \varphi & & \text { weak* in } L^{\infty}\left(0, T ; H^{1}(\Omega)\right), \text { strongly in } L^{q_{2}}\left(\Omega_{T}\right) ; \\
\varepsilon^{2} \partial_{t} \varphi_{\varepsilon} & \longrightarrow 0 & & \text { strongly in } L^{2}\left(\Omega_{T}\right) ; \\
\varepsilon^{2} \nabla \varphi_{\varepsilon} & \longrightarrow 0 & & \text { strongly in } L^{\infty}\left(0, T ;\left[L^{2}(\Omega)\right]^{d}\right) ; \\
s_{\varepsilon} \partial_{t} \varphi_{\varepsilon} & \longrightarrow \psi & & \text { weakly in } L^{2}\left(\Omega_{T}\right) ; \\
s_{\varepsilon} \nabla \varphi_{\varepsilon} & \longrightarrow \chi & & \text { weak* in } L^{\infty}\left(0, T ;\left[L^{2}(\Omega)\right]^{d}\right) ;
\end{aligned}
$$

for some $\psi \in L^{2}\left(\Omega_{T}\right)$ and $\chi \in L^{\infty}\left((0, T) ;\left[L^{2}(\Omega)\right]^{d}\right)$; where $q_{i} \in[1, \infty)$, but if either (a) $d=1$ or (b) $\delta>0$ then $q_{1} \in[1, \infty]$. Once again, for brevity, in the above we adopt the same notation for the subsequence.

Step 2 (identifying $\chi$ and $\psi$, and passing to the limit in (2.4b)). We now show that

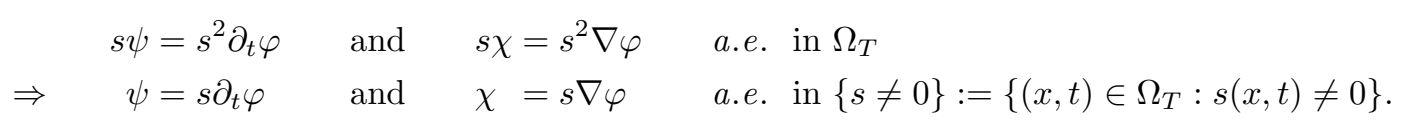

This will be achieved by using the definition, and uniqueness, of weak derivatives. For any $w \in\left[C_{0}^{1}\left(\Omega_{T}\right)\right]^{d}$, integration by parts yields that

$$
-\int_{\Omega_{T}} s_{\varepsilon}^{2} \varphi_{\varepsilon} \operatorname{div} w \mathrm{~d} x \mathrm{~d} t=\int_{\Omega_{T}} \nabla\left(s_{\varepsilon}^{2} \varphi_{\varepsilon}\right) \cdot w \mathrm{~d} x \mathrm{~d} t=\int_{\Omega_{T}}\left(2 s_{\varepsilon} \varphi_{\varepsilon} \nabla s_{\varepsilon}+s_{\varepsilon}^{2} \nabla \varphi_{\varepsilon}\right) \cdot w \mathrm{~d} x \mathrm{~d} t .
$$

Letting $\varepsilon \rightarrow 0$ and noting (2.45), we obtain that

$$
-\int_{\Omega_{T}} s^{2} \varphi \operatorname{div} w \mathrm{~d} x \mathrm{~d} t=\int_{\Omega_{T}}(2 s \varphi \nabla s+s \chi) \cdot w \mathrm{~d} x \mathrm{~d} t .
$$

Hence we have that

$$
\nabla\left(s^{2} \varphi\right)=\varphi \nabla\left(s^{2}\right)+s \chi \quad \text { or } \quad s \chi=\nabla\left(s^{2} \varphi\right)-\varphi \nabla\left(s^{2}\right)=s^{2} \nabla \varphi
$$

which implies the desired result on $\chi$ in (2.46). Similarly, to the above we can show the desired result on $\psi$ in (2.46). Noting (2.45) and (2.46), we can now pass to the limit $\varepsilon \rightarrow 0$ in a subsequence of (2.4b) to obtain (2.41b).

Step 3 (strong convergence of $\left.s_{\varepsilon} \nabla \varphi_{\varepsilon}\right)$. Similarly to (2.22), we choose $\eta=\varphi_{\varepsilon}-\phi \in L^{2}\left(0, T ; H^{1}(\Omega)\right)$ in (2.4b) and obtain from (2.45) and (2.46) that

$$
\begin{aligned}
\limsup _{\varepsilon \rightarrow 0} \int_{\Omega_{T}}\left(\left(s_{\varepsilon}\right)^{2}+\varepsilon^{2}\right)\left|\nabla \varphi_{\varepsilon}\right|^{2} \mathrm{~d} x \mathrm{~d} t & =\limsup _{\varepsilon \rightarrow 0} \int_{\Omega_{T}}\left(\left(s_{\varepsilon}\right)^{2}+\varepsilon^{2}\right)\left[\nabla \varphi_{\varepsilon} \cdot \nabla \phi-k_{3}^{-1} \partial_{t} \varphi_{\varepsilon}\left(\varphi_{\varepsilon}-\phi\right)\right] \mathrm{d} x \mathrm{~d} t \\
& =\int_{\{s \neq 0\}} s^{2}\left[\nabla \varphi \cdot \nabla \phi-k_{3}^{-1} \partial_{t} \varphi(\varphi-\phi)\right] \mathrm{d} x \mathrm{~d} t
\end{aligned}
$$

Once again similarly to (2.22), we would like to choose $\eta=\varphi-\phi$ in (2.41b) to obtain the desired result. Unfortunately, this choice is not justified as $\varphi \notin L^{2}\left(0, T ; H^{1}(\Omega)\right)$. However, this problem can be overcome by 
applying the following technique adapted from [7]. Firstly, we note that

$$
\int_{0}^{1} q^{-1} \mathrm{~d} q=\infty \quad \Rightarrow \quad \forall \sigma>0, \exists \text { a unique } \mu(\sigma) \in(0, \sigma) \text { s.t. } \int_{\mu}^{\sigma} q^{-1} \mathrm{~d} q=1
$$

Let $f_{\mu, \sigma}$ be defined by

$$
f_{\mu, \sigma}(r):=\left\{\begin{array}{ll}
1 & \text { if }|r| \geq \sigma \\
\int_{\mu}^{r} q^{-1} \mathrm{~d} q & \text { if }|r| \in[\mu, \sigma], \\
0 & \text { if }|r| \leq \mu
\end{array} \quad \Rightarrow \quad f_{\mu, \sigma}^{\prime}(r)= \begin{cases}0 & \text { if }|r|>\sigma \\
r^{-1} & \text { if }|r| \in(\mu, \sigma), . \\
0 & \text { if }|r|<\mu\end{cases}\right.
$$

As $s \nabla \varphi \in\left[L^{2}(s \neq 0)\right]^{d}$, recall $(2.45)$ and (2.46)), it is easily established for all $\sigma>0$ that $\eta \equiv f_{\mu, \sigma}(s)(\varphi-\phi) \in$ $L^{2}\left(0, T ; H_{0}^{1}(\Omega)\right)$. Choosing such an $\eta$ in $(2.41 \mathrm{~b})$ and recalling $(2.50)$, we obtain that

$$
\begin{aligned}
\int_{\{s \neq 0\}} s^{2}\left[k_{3}^{-1} \partial_{t}\right. & \varphi(\varphi-\phi)+\nabla \varphi \cdot \nabla(\varphi-\phi)] \mathrm{d} x \mathrm{~d} t \\
& =\lim _{\sigma \rightarrow 0} \int_{\{s \neq 0\}} s^{2} f_{\mu, \sigma}(s)\left[k_{3}^{-1} \partial_{t} \varphi(\varphi-\phi)+\nabla \varphi \cdot \nabla(\varphi-\phi)\right] \mathrm{d} x \mathrm{~d} t \\
& =-\lim _{\sigma \rightarrow 0} \int_{\{\mu<|s|<\sigma\} \cap\{s \neq 0\}} s \nabla \varphi \cdot \nabla s(\varphi-\phi) \mathrm{d} x \mathrm{~d} t=0 .
\end{aligned}
$$

Noting (2.45), and combining (2.48) and (2.51) yields that

$$
\int_{\Omega_{T}}|\chi|^{2} \mathrm{~d} x \mathrm{~d} t \leq \limsup _{\varepsilon \rightarrow 0} \int_{\Omega_{T}}\left(\left(s_{\varepsilon}\right)^{2}+\varepsilon^{2}\right)\left|\nabla \varphi_{\varepsilon}\right|^{2} \mathrm{~d} x \mathrm{~d} t=\int_{\{s \neq 0\}} s^{2}|\nabla \varphi|^{2} \mathrm{~d} x \mathrm{~d} t .
$$

Similarly to (2.23), we obtain from $(2.45),(2.46)$ and $(2.52)$ that

$$
\limsup _{\varepsilon \rightarrow 0} \int_{\Omega_{T}}\left|s_{\varepsilon} \nabla \varphi_{\varepsilon}-\chi\right|^{2} \mathrm{~d} x \mathrm{~d} t \leq \int_{\{s \neq 0\}} s^{2}|\nabla \varphi|^{2} \mathrm{~d} x \mathrm{~d} t-\int_{\Omega_{T}}|\chi|^{2} \mathrm{~d} x \mathrm{~d} t=0 .
$$

Combining (2.53), (2.46) and (2.52) it follows that

$$
s_{\varepsilon} \nabla \varphi_{\varepsilon} \longrightarrow \chi=\left\{\begin{array}{ll}
s \nabla \varphi & s \neq 0 \\
0 & s=0
\end{array} \quad \text { strongly in } L^{2}\left(0, T ;\left[L^{2}(\Omega)\right]^{d}\right) \text { as } \varepsilon \rightarrow 0 .\right.
$$

Step 4 (passing to the limit in $(2.4 \mathrm{a})$ with $v$ replaced by $\left.s_{\varepsilon} v\right)$. Since $(2.41 \mathrm{~b})$ has already been verified, it remains to show $\{s, \varphi\}$ also satisfies (2.41a). To this end, for any $\sigma>0$ let $b_{\sigma}, \omega_{\sigma} \in C^{1}(\mathbb{R})$ be defined as

$$
\begin{aligned}
& b_{\sigma}(r):= \begin{cases}0 & \text { if } r \in[0, \sigma], \\
\sigma\left[5\left(\frac{r-\sigma}{\sigma}\right)^{2}-3\left(\frac{r-\sigma}{\sigma}\right)^{3}\right] & \text { if } r \in[\sigma, 2 \sigma], \quad \text { and } \quad b_{\sigma}(-r)=-b_{\sigma}(r) \quad \forall r \geq 0 \\
r & \text { if } r \geq 2 \sigma\end{cases} \\
& \omega_{\sigma}(r):= \begin{cases}0 & \text { if }|r| \leq \sigma, \\
\sigma\left[3\left(\frac{r-\sigma}{\sigma}\right)^{2}-2\left(\frac{r-\sigma}{\sigma}\right)^{3}\right] & \text { if }|r| \in[\sigma, 2 \sigma], . \\
1 & \text { if }|r| \geq 2 \sigma\end{cases}
\end{aligned}
$$

We note that

$$
0 \leq b_{\sigma}(r) \leq r \leq b_{\sigma}(r)+2 \sigma \quad \forall r \geq 0, \forall \sigma>0 \quad \text { and } \quad \text { for } r \neq 0 \quad b_{\sigma}^{\prime}(r) \rightarrow 1 \quad \text { as } \quad \sigma \rightarrow 0
$$


If either (a) $\delta \geq 0$ and $p>1$ if $d=1$ or (b) $\delta>0$ and $p>d$ if $d \geq 2$, then we have from (2.45) and (2.54) that as $\varepsilon \rightarrow 0$

$$
s_{\varepsilon} \longrightarrow s \quad \text { strongly in } L^{\infty}\left(\Omega_{T}\right) \quad \Rightarrow \quad \nabla \varphi_{\varepsilon} \longrightarrow \nabla \varphi \text { strongly in }\left[L^{2}(\{|s|>\sigma\})\right]^{d} \text { for any } \sigma>0 .
$$

We now pass to the limit $\varepsilon \rightarrow 0$ in (2.4a) with $v$ replaced by $s_{\varepsilon} v$ by adapting the approach in (2.26) and (2.27). Firstly we have for all $\sigma>0, \lambda \in \mathbb{R}$ and for all $v \in L^{2}\left((0, T) ; W_{0}^{1, p^{\star}}(\Omega) \cap L^{\infty}(\Omega)\right)$ that

$0 \leq k_{1} \int_{\Omega_{T}} \omega_{\frac{\sigma}{2}}(s)\left\{\left[1+\delta\left|\nabla\left(s+\lambda b_{\sigma}(s) v\right)\right|^{p-2}\right] \nabla\left(s+\lambda b_{\sigma}(s) v\right)-\left[1+\delta\left|\nabla s_{\varepsilon}\right|^{p-2}\right] \nabla s_{\varepsilon}\right\} \cdot \nabla\left[s+\lambda b_{\sigma}(s) v-s_{\varepsilon}\right] \mathrm{d} x \mathrm{~d} t$.

Next from (2.4a) and (2.55a,b) we note for all $\sigma>0, \lambda \in \mathbb{R}$ and for all $v \in L^{2}\left((0, T) ; W_{0}^{1, p^{\star}}(\Omega) \cap L^{\infty}(\Omega)\right)$ that

$$
\begin{gathered}
-k_{1} \int_{\Omega_{T}} \omega_{\frac{\sigma}{2}}(s)\left[1+\delta\left|\nabla s_{\varepsilon}\right|^{p-2}\right] \nabla s_{\varepsilon} \cdot \nabla\left[s+\lambda b_{\sigma}(s) v-s_{\varepsilon}\right] \mathrm{d} x \mathrm{~d} t \\
=-k_{1} \int_{\Omega_{T}}\left[1+\delta\left|\nabla s_{\varepsilon}\right|^{p-2}\right] \nabla s_{\varepsilon} \cdot\left\{\nabla\left[\omega_{\frac{\sigma}{2}}(s)\left(s+\lambda b_{\sigma}(s) v-s_{\varepsilon}\right)\right]-\left[\left(s+\lambda b_{\sigma}(s) v-s_{\varepsilon}\right) \nabla \omega_{\frac{\sigma}{2}}(s)\right]\right\} \mathrm{d} x \mathrm{~d} t \\
=\int_{\Omega_{T}} \omega_{\frac{\sigma}{2}}(s)\left[\partial_{t} s_{\varepsilon}+k_{2}\left|\nabla \varphi_{\varepsilon}\right|^{2} s_{\varepsilon}+W^{\prime}\left(s_{\varepsilon}\right)\right]\left(s+\lambda b_{\sigma}(s) v-s_{\varepsilon}\right) \mathrm{d} x \mathrm{~d} t \\
-k_{1} \int_{\Omega_{T}}\left[1+\delta\left|\nabla s_{\varepsilon}\right|^{p-2}\right] \nabla s_{\varepsilon} \cdot\left[\left(s-s_{\varepsilon}\right) \nabla \omega_{\frac{\sigma}{2}}(s)\right] \mathrm{d} x \mathrm{~d} t .
\end{gathered}
$$

On noting (2.45), (2.54), (2.55a,b) and (2.57), we now pass to the limit $\varepsilon \rightarrow 0$ in (2.58) and (2.59), and on combining yields for all $\sigma>0, \lambda \in \mathbb{R}$ and for all $v \in L^{2}\left((0, T) ; W_{0}^{1, p^{*}}(\Omega) \cap L^{\infty}(\Omega)\right)$ that

$$
\begin{aligned}
0 \leq \lambda k_{1} \int_{\Omega_{T}}\left[1+\delta\left|\nabla\left(s+\lambda b_{\sigma}(s) v\right)\right|^{p-2}\right] \nabla\left(s+\lambda b_{\sigma}(s) v\right) & \cdot \nabla\left(b_{\sigma}(s) v\right) \mathrm{d} x \mathrm{~d} t \\
& +\lambda \int_{\Omega_{T}}\left[\partial_{t} s+k_{2} \chi \cdot \nabla \varphi+W^{\prime}(s)\right] b_{\sigma}(s) v \mathrm{~d} x \mathrm{~d} t .
\end{aligned}
$$

On noting (2.56), $|\nabla s|=0$ if $s=0$, and a generalised Lebesgue dominated convergence theorem, we now pass to the limit $\sigma \rightarrow 0$ in (2.60), which yields for all $\lambda \in \mathbb{R}$ and for all $v \in L^{2}\left((0, T) ; W_{0}^{1, p^{\star}}(\Omega) \cap L^{\infty}(\Omega)\right)$ that

$$
0 \leq \lambda\left[\int_{\Omega_{T}}\left\{k_{1}\left[1+\delta|\nabla(s+\lambda s v)|^{p-2}\right] \nabla(s+\lambda s v) \cdot \nabla(s v) \mathrm{d} x \mathrm{~d} t+\left[s \partial_{t} s+k_{2}|\chi|^{2}+s W^{\prime}(s)\right] v\right\} \mathrm{d} x \mathrm{~d} t\right] .
$$

Considering the cases of $\lambda>0$ and $\lambda<0$ separately, we divide (2.61) by $\lambda$ and then pass to the limit $\lambda \rightarrow 0$ to obtain the desired result (2.41a) on noting (2.54).

Finally, similarly to (2.28), it follows from (2.8a,b), (2.42b), (2.45), (2.54) and (2.13a) that for a.a. $\tilde{t} \in(0, T)$

$$
\begin{aligned}
& E(s, \varphi)(\tilde{t})+\int_{\Omega_{\tilde{t}}}\left\{\left(\partial_{t} s\right)^{2}+\frac{k_{2}}{k_{3}}\left(s \partial_{t} \varphi\right)^{2}\right\} \mathrm{d} x \mathrm{~d} t \\
& \quad \leq \liminf _{\varepsilon \rightarrow 0}\left[E_{\varepsilon}\left(s_{\varepsilon}, \varphi_{\varepsilon}\right)(\tilde{t})+\int_{\Omega_{\tilde{t}}}\left\{\left(\partial_{t} s_{\varepsilon}\right)^{2}+\frac{k_{2}}{k_{3}}\left(\left(s_{\varepsilon}\right)^{2}+\varepsilon^{2}\right)\left(\partial_{t} \varphi_{\varepsilon}\right)^{2}\right\} \mathrm{d} x \mathrm{~d} t\right] \leq \lim _{\varepsilon \rightarrow 0} E_{\varepsilon}(g, \phi)=E(g, \phi),
\end{aligned}
$$

and hence the desired dissipative energy law (2.42a). 
We conclude this section by the following remark.

Remark 2.10. For $d \geq 2$ and $\delta=0$, we are only able to establish that a subsequence $s_{\varepsilon} \rightarrow s$ strongly in $L^{q_{1}}\left(\Omega_{T}\right)$ with $q_{1} \in[1, \infty)$, recall (2.45). In order to pass to the limit $\varepsilon \rightarrow 0$ on the term involving $\left|\nabla \varphi_{\varepsilon}\right|^{2}$ in (2.4a) with $v$ replaced by $s_{\varepsilon} v$, we require $s_{\varepsilon} \rightarrow s$ strongly in $L^{\infty}\left(\Omega_{T}\right)$, recall (2.57). This is the only reason for our $p$-Laplacian modification, $\left(\mathrm{P}_{\delta}\right)$, of $\left(\mathrm{P}_{0}\right)$. Although we are not aware of any physical justification for the inclusion of this $p$-Laplacian term, we would like to stress that its inclusion does not effect the essential features of the degenerate system $\left(\mathrm{P}_{0}\right)$. We note that it is only Step 4 of the proof of Theorem 2.9 that requires the restriction $\delta>0$ with $p>d$. Hence for $\delta=0$, we still have weak* convergence of a subsequence $s_{\varepsilon}$ to $s$ in $L^{\infty}\left(\Omega_{T}\right)$, and that $s \in\left[g_{-}, g_{+}\right]$. So the addition of this $p$-Laplacian term does not strengthen the control of $s$ itself. In addition, the inclusion of such a term does not require additional artificial boundary conditions as would be the case if we circumvented the mathematical difficulty above by introducing, instead of the $p$ Laplacian term $\delta \operatorname{div}\left(|\nabla s|^{p-2} \nabla s\right)$, the fourth order linear term $-\delta \Delta^{2} s$ in (1.1a). Moreover, $\left(\mathrm{P}_{\delta}\right)$ satisfies a very similar Lyapunov structure to $\left(\mathrm{P}_{0}\right)$ as the energy $E(s, \varphi)$ still only depends on $|\nabla s|, W(s)$ and $|s \nabla \varphi|$; recall $(2.42 \mathrm{a}, \mathrm{b})$.

Finally, it should be noted that the results, and proofs, of Theorems 2.4, 2.5 and Corollary 2.6 remain valid when the restriction $p>d$ is weakened to $p>1$.

\section{Convergence of the finite Element approximation}

In this section, we present a practical fully discrete finite element method for approximating the regularized problem $\left(\mathrm{P}_{\delta, \epsilon}\right),(2.4 \mathrm{a}, \mathrm{b})$, and the degenerate problem $\left(\mathrm{P}_{\delta}\right),(2.41 \mathrm{a}, \mathrm{b})$. Establishing subsequence convergence of the approximation to (i) $\left(\mathrm{P}_{\delta, \epsilon}\right)$, with $\delta \geq 0, p>d$ and $\varepsilon \in(0,1]$, as both the spatial mesh size parameter, $h$, and the time step parameter, $\tau$, tend to zero with fixed $\varepsilon \in(0,1]$; and (ii) $\left(\mathrm{P}_{\delta}\right)$, with either (a) $\delta=0$ if $d=1$ or (b) $\delta>0$ and $p>d$ if $d \geq 1$, as $h, \tau$ and $\varepsilon$ all tend to zero. The key elements for the convergence are to establish a discrete energy law and a discrete maximum principle which mimic the energy law $(2.8 \mathrm{a}, \mathrm{b})$ and the maximum principle, (ii) of Definition 2.1, for $\left(\mathrm{P}_{\delta, \varepsilon}\right)$.

\subsection{Fully discrete finite element method}

For ease of exposition, we will assume that $\Omega$ is polygonal if $d=2$ and polyhedral if $d=3$. Let $\left\{\mathcal{T}^{h}\right\}_{h}$ be a regular family of partitionings of $\Omega$ into disjoint open simplices $K$ with $h_{K}:=\operatorname{diam}(K)$ and $h:=\max _{K \in \mathcal{T}^{h}} h_{K}$, so that $\bar{\Omega}=\cup_{K \in \mathcal{T}^{h}} \bar{K}$. In addition, it is assumed that $\mathcal{T}^{h}$ is a (weakly) acute partitioning; that is for (a) $d=2$, for any pair of adjacent triangles the sum of opposite angles relative to the common side does not exceed $\pi$; (b) $d=3$, the angle between any faces of the same tetrahedron does not exceed $\frac{\pi}{2}$.

Let $V^{h}$ denote the finite element space of continuous, piecewise linear functions associated with $\mathcal{T}^{h}$; that is,

$$
V^{h}:=\left\{v^{h} \in C^{0}(\bar{\Omega}) ;\left.v^{h}\right|_{K} \in P_{1}, \forall K \in \mathcal{T}^{h}\right\} \quad \text { and } \quad V_{0}^{h}:=V^{h} \cap H_{0}^{1}(\Omega),
$$

where $P_{1}$ is the set of linear polynomials in $d$ variables. Let $J$ be the set of nodes of $\mathcal{T}^{h}$ and $\left\{p_{j}\right\}_{j \in J}$ the coordinates of these nodes. Let $\left\{\chi_{j}\right\}_{j \in J}$ be the standard basis functions for $V^{h}$; that is $\chi_{j} \in V^{h}$ and $\chi_{j}\left(p_{i}\right)=\delta_{i j}$ for all $i, j \in J$. We introduce $\pi^{h}: C(\bar{\Omega}) \rightarrow V^{h}$, the interpolation operator, such that $\left(\pi^{h} \eta\right)\left(p_{j}\right)=\eta\left(p_{j}\right)$ for all $j \in J$. A discrete semi-inner product on $C(\bar{\Omega})$ is then defined by

$$
\left(\eta_{1}, \eta_{2}\right)^{h}:=\int_{\Omega} \pi^{h}\left[\eta_{1}(x) \eta_{2}(x)\right] \mathrm{d} x .=\sum_{j \in J} \omega_{j} \eta_{1}\left(p_{j}\right) \eta_{2}\left(p_{j}\right), \quad \text { where } \quad \omega_{j}:=\left(1, \chi_{j}\right)>0 .
$$

For convenience, we will assume that $g, \phi \in W^{1, r^{\star}}(\Omega)$, where $r^{\star}:=\max \left\{r, p^{\star}\right\}$ for some $r>d$, so that one can set

$$
V_{g}^{h}:=\left\{v^{h} \in V^{h}: v^{h}-\pi^{h} g \in V_{0}^{h}\right\} \quad \text { and } \quad V_{\phi}^{h}:=\left\{\eta^{h} \in V^{h}: \eta^{h}-\pi^{h} \phi \in V_{0}^{h}\right\} .
$$


Adopting the same notation as used in $\left(\mathrm{P}_{\delta, \varepsilon}^{\tau}\right),(2.7 \mathrm{a}, \mathrm{b})$; our fully discrete finite element approximation of $\left(\mathrm{P}_{\delta, \varepsilon}\right)$ is then:

$\left(\mathbf{P}_{\delta, \varepsilon}^{h, \tau}\right)$ Let $\left\{S_{\varepsilon}^{0}, \Phi_{\varepsilon}^{0}\right\}=\left\{\pi^{h} g, \pi^{h} \phi\right\}$, then for $m=1,2, \cdots M$, find $S_{\varepsilon}^{m} \in V_{g}^{h}$ and $\Phi_{\varepsilon}^{m} \in V_{\phi}^{h}$ such that

$$
\begin{aligned}
\left(d_{t} S_{\varepsilon}^{m}, v^{h}\right)^{h}+k_{1}\left(\left[1+\delta\left|\nabla S_{\varepsilon}^{m}\right|^{p-2}\right] \nabla S_{\varepsilon}^{m}, \nabla v^{h}\right)+k_{2}\left(\left|\nabla \Phi_{\varepsilon}^{m-1}\right|^{2}, \pi^{h}\left[S_{\varepsilon}^{m} v^{h}\right]\right)+\left(\widetilde{W}_{+}^{\prime}\left(S_{\varepsilon}^{m}\right), v^{h}\right)^{h} & \\
=\left(\widetilde{W}_{-}^{\prime}\left(S_{\varepsilon}^{m-1}\right), v^{h}\right)^{h} & \forall v^{h} \in V_{0}^{h}, \\
\left(\left[\left(S_{\varepsilon}^{m}\right)^{2}+\varepsilon^{2}\right] d_{t} \Phi_{\varepsilon}^{m}, \eta^{h}\right)^{h}+k_{3}\left(\pi^{h}\left[\left(S_{\varepsilon}^{m}\right)^{2}+\varepsilon^{2}\right] \nabla \Phi_{\varepsilon}^{m}, \nabla \eta^{h}\right)=0 & \forall \eta^{h} \in V_{0}^{h} .
\end{aligned}
$$

The above finite element method is a semi-implicit scheme. For $m=1,2, \cdots M$, given $S_{\varepsilon}^{m-1} \in V_{g}^{h}$ and $\Phi_{\varepsilon}^{m-1} \in$ $V_{\phi}^{h}$ one first solves the resulting semi-linear system, (3.3a), with a diagonal monotonically increasing nonlinearity, for $S_{\varepsilon}^{m}$; then the linear system $(3.3 \mathrm{~b})$ for $\Phi_{\varepsilon}^{m}$. Adopting similar notation to that used in $(2.17 \mathrm{a}, \mathrm{b}),\left(\mathrm{P}_{\delta, \varepsilon}^{h, \tau}\right)$ can be restated as: Find $\left\{S_{\varepsilon}, \Phi_{\varepsilon}\right\} \in C\left([0, T] ; V_{g}^{h}\right) \times C\left([0, T] ; V_{\phi}^{h}\right)$ such that $S_{\varepsilon}(\cdot, 0)=\left(\pi^{h} g\right)(\cdot), \Phi_{\varepsilon}(\cdot, 0)=\left(\pi^{h} \phi\right)(\cdot)$ and

$$
\begin{aligned}
\int_{0}^{T}\left\{\left(\partial_{t} S_{\varepsilon}, v^{h}\right)^{h}+k_{1}\left(\left[1+\delta\left|\nabla S_{\varepsilon}^{+}\right|^{p-2}\right] \nabla S_{\varepsilon}^{+}, \nabla v^{h}\right)+k_{2}\left(\left|\nabla \Phi_{\varepsilon}^{-}\right|^{2}, \pi^{h}\left[S_{\varepsilon}^{+} v^{h}\right]\right)\right. & \\
\left.+\left(\left[\widetilde{W}_{+}^{\prime}\left(S_{\varepsilon}^{+}\right)-\widetilde{W}_{-}^{\prime}\left(S_{\varepsilon}^{-}\right)\right], v^{h}\right)^{h}\right\} \mathrm{d} t=0 & \forall v^{h} \in L^{2}\left(0, T ; V_{0}^{h}\right), \\
\int_{0}^{T}\left\{\left(\left[\left(S_{\varepsilon}^{+}\right)^{2}+\varepsilon^{2}\right] \partial_{t} \Phi_{\varepsilon}, \eta^{h}\right)^{h}+k_{3}\left(\pi^{h}\left[\left(S_{\varepsilon}^{+}\right)^{2}+\varepsilon^{2}\right] \nabla \Phi_{\varepsilon}^{+}, \nabla \eta^{h}\right)\right\} \mathrm{d} t=0 & \forall \eta^{h} \in L^{2}\left(0, T ; V_{0}^{h}\right) .
\end{aligned}
$$

We end this subsection by recalling some well-known results concerning $V^{h}$. For any $K \in \mathcal{T}^{h}, v^{h}, \eta^{h} \in V^{h}$, and $m \in\{0,1\}$ :

$$
\begin{array}{rlrl}
\lim _{h \rightarrow 0}\left\|\left(I-\pi^{h}\right) \eta\right\|_{W^{1, r}} & =0 & \forall \eta \in W^{1, r}(\Omega), r>d ; \\
\int_{K}\left(v^{h}\right)^{2} \mathrm{~d} x & \leq \int_{K} \pi^{h}\left[\left(v^{h}\right)^{2}\right] \mathrm{d} x \leq(d+2) \int_{K}\left(v^{h}\right)^{2} \mathrm{~d} x ; & \\
\left|\int_{K}\left(I-\pi^{h}\right)\left[v^{h} \eta^{h}\right] \mathrm{d} x\right| & \leq\left\|\left(I-\pi^{h}\right)\left(v^{h} \eta^{h}\right)\right\|_{L^{1}(K)} \leq C h_{K}^{2-m}\left|v^{h}\right|_{H^{1}(K)}\left|\eta^{h}\right|_{H^{1-m}(K)} ; \\
\left\|\left(I-\pi^{h}\right)\left[v^{h} \eta^{h}\right]\right\|_{W^{m, r}(K)} & \leq C h_{K}^{2-m-1}\left\|v^{h}\right\|_{L^{\infty}(K)}\left\|\nabla \eta^{h}\right\|_{L^{r}(K)} & r \in[1, \infty] .
\end{array}
$$

We note that the (weak) acuteness assumption on $\mathcal{T}^{h}$ yields that

$$
\int_{K} \nabla \chi_{i} \cdot \nabla \chi_{j} \mathrm{~d} x \leq 0 \quad i \neq j, \quad \forall K \in \mathcal{T}^{h} .
$$

Finally let $f \in C^{0,1}(\mathbb{R})$ be monotone with Lipschitz constant $L_{f}$, then it follows from (3.9) and the inequality

$$
(f(a)-f(b))^{2} \leq L_{f}(f(a)-f(b))(a-b) \quad \forall a, b \in \mathbb{R}
$$

that for all $v^{h} \in V^{h}$

$$
\int_{K}\left|\nabla \pi^{h}\left[f\left(v^{h}\right)\right]\right|^{2} \mathrm{~d} x \leq L_{f} \int_{K} \nabla v^{h} \cdot \nabla \pi^{h}\left[f\left(v^{h}\right)\right] \mathrm{d} x \quad \forall K \in \mathcal{T}^{h} .
$$

\subsection{Convergence of $\left(\mathbf{P}_{\delta, \varepsilon}^{h, \tau}\right)$ for fixed $\varepsilon>0$ as $h, \tau \rightarrow 0$}

In this subsection, we study the limiting behaviour of the finite element solution $\left\{S_{\varepsilon}, \Phi_{\varepsilon}\right\}$ of $\left(\mathrm{P}_{\delta, \varepsilon}^{h, \tau}\right)$ as $h, \tau \rightarrow 0$ for a fixed $\varepsilon>0$. We having the following analogue of Theorem 2.4. 
Theorem 3.1. For any fixed $\delta \geq 0, p>d$ and $\varepsilon \in(0,1]$, there exits a unique solution $\left\{S_{\varepsilon}, \Phi_{\varepsilon}\right\}$ to the system $\left(P_{\delta, \varepsilon}^{h, \tau}\right)$. In addition, it follows that

$$
S_{\varepsilon}^{( \pm)} \in\left[g_{-}, g_{+}\right] \quad \text { and } \quad \Phi_{\varepsilon}^{( \pm)} \in\left[\phi_{-}, \phi_{+}\right]
$$

Furthermore, it satisfies the following dissipative energy law for all $\tilde{t} \in(0, T]$

$$
\begin{array}{r}
E_{\varepsilon}^{h}\left(S_{\varepsilon}^{+}, \Phi_{\varepsilon}^{+}\right)(\tilde{t})+\int_{\Omega_{\tilde{t}}} \bar{\tau}\left\{\frac{k_{1}}{2}\left|\nabla\left(\partial_{t} S_{\varepsilon}\right)\right|^{2}+\frac{k_{2}}{2}\left(\pi^{h}\left[\left(\partial_{t} S_{\varepsilon}\right)^{2}\right]\left|\nabla \Phi_{\varepsilon}^{-}\right|^{2}+\left.\pi^{h}\left[\left(S_{\varepsilon}^{+}\right)^{2}+\varepsilon^{2}\right] \nabla\left(\partial_{t} \Phi_{\varepsilon}\right)\right|^{2}\right)\right\} \mathrm{d} x \mathrm{~d} t \\
+\int_{\Omega_{\tilde{t}}} \pi^{h}\left[\left(\partial_{t} S_{\varepsilon}\right)^{2}+\frac{k_{2}}{k_{3}}\left(\left(S_{\varepsilon}^{+}\right)^{2}+\varepsilon^{2}\right)\left(\partial_{t} \Phi_{\varepsilon}\right)^{2}\right] \mathrm{d} x \mathrm{~d} t \leq E_{\varepsilon}^{h}\left(\pi^{h} g, \pi^{h} \phi\right) \leq C
\end{array}
$$

where $C$ is independent of $h, \tau$ and $\varepsilon, \bar{\tau}(t):=\tau_{m}$ if $t \in\left(t_{m-1}, t_{m}\right], m=1,2, \cdots M$, and

$E_{\varepsilon}^{h}\left(S_{\varepsilon}, \Phi_{\varepsilon}\right)(t):=\int_{\Omega}\left\{k_{1}\left[\frac{1}{2}\left|\nabla S_{\varepsilon}(x, t)\right|^{2}+\frac{\delta}{p}\left|\nabla S_{\varepsilon}(x, t)\right|^{p}\right]+\frac{k_{2}}{2} \pi^{h}\left[S_{\varepsilon}^{2}+\varepsilon^{2}\right](x, t)\left|\nabla \Phi_{\varepsilon}(x, t)\right|^{2}+\pi^{h}\left[W\left(S_{\varepsilon}\right)\right](x, t)\right\} \mathrm{d} x$.

Moreover, there exists a subsequence $\left\{S_{\varepsilon}^{( \pm)}, \Phi_{\varepsilon}^{( \pm)}\right\}_{h, \tau>0}$ such that as $h, \tau \rightarrow 0$

$\partial_{t} S_{\varepsilon} \longrightarrow \partial_{t} s_{\varepsilon} \quad$ weakly in $L^{2}\left(\Omega_{T}\right), \quad S_{\varepsilon}^{( \pm)} \longrightarrow s_{\varepsilon} \quad$ weak ${ }^{*}$ in $L^{\infty}\left(0, T ; W^{1, p^{*}}(\Omega)\right), \quad$ strongly in $L^{q_{1}}\left(\Omega_{T}\right)$,

$\partial_{t} \Phi_{\varepsilon} \longrightarrow \partial_{t} \varphi_{\varepsilon} \quad$ weakly in $L^{2}\left(\Omega_{T}\right), \quad \Phi_{\varepsilon}^{( \pm)} \longrightarrow \varphi_{\varepsilon} \quad$ weak ${ }^{*}$ in $L^{\infty}\left(0, T ; H^{1}(\Omega)\right), \quad$ strongly in $L^{q_{2}}\left(\Omega_{T}\right)$;

where $\left\{s_{\varepsilon}, \varphi_{\varepsilon}\right\}$ is a weak solution to $\left(P_{\delta, \varepsilon}\right)$ and $q_{i} \in[1, \infty)$, but if either $(a) d=1$ or (b) $\delta>0$ then $q_{1} \in[1, \infty]$.

Proof. The proof is similar to that of Theorem 2.4. We just stress the main differences. It is easily established that there exists a unique solution $\left\{S_{\varepsilon}^{m}, \Phi_{\varepsilon}^{m}\right\}, m=1,2, \cdots M$, to (3.3a,b). Hence there exists a unique solution $\left\{S_{\varepsilon}, \Phi_{\varepsilon}\right\}$ to $\left(\mathrm{P}_{\delta, \varepsilon}^{h, \tau}\right)$. Assuming $S_{\varepsilon}^{m-1} \in\left[g_{-}, g_{+}\right], \Phi_{\varepsilon}^{m-1} \in\left[\phi_{-}, \phi_{+}\right]$, noting that it is true for $m=1$, and choosing $v^{h}=\pi^{h}\left[S_{\varepsilon}^{m}-g_{+}\right]_{+}, \pi^{h}\left[S_{\varepsilon}^{m}-g_{-}\right]_{-} \in V_{0}^{h}$ in (3.3a), $\eta^{h}=\pi^{h}\left[\Phi_{\varepsilon}^{m}-\phi_{+}\right]_{+}, \pi^{h}\left[\Phi_{\varepsilon}^{m}-\phi_{-}\right]_{-} \in V_{0}^{h}$ in (3.3b) with the obvious adaptation of the argument in (2.10), on noting (3.10), yields the desired result (3.11). On choosing $v^{h}=d_{t} S_{\varepsilon}^{m}$ in (3.3a) and $\eta^{h}=\frac{k_{2}}{k_{3}} d_{t} \Phi_{\varepsilon}^{m}$ in (3.3b), and adding yields, similarly to (2.15), the desired result (3.12a,b), on noting (3.5).

Similarly to (2.20), the energy law (3.12a,b) yields, on noting a Poincaré inequality, and (3.6), the subsequence convergence results (3.13). Once again for brevity, we adopt the same notation for the subsequence throughout. Obviously, we still need to establish that the limit $\left\{s_{\varepsilon}, \varphi_{\varepsilon}\right\}$ is a weak solution of $\left(\mathrm{P}_{\delta, \varepsilon}\right)$. Clearly, (3.11), (3.13) and (3.5) yield that (i)-(iv) of Definition 2.1 hold. We now need to show that $\left\{s_{\varepsilon}, \varphi_{\varepsilon}\right\}$ satisfy $(2.4 \mathrm{a}, \mathrm{b})$.

Similarly to (2.21), it follows from (3.13), (3.11), (3.8), (3.7) and (3.12a,b) on extracting a further subsequence that as $h, \tau \rightarrow 0$

$$
\begin{aligned}
\pi^{h}\left[\left(\left(S_{\varepsilon}^{+}\right)^{2}+\varepsilon^{2}\right) \partial_{t} \Phi_{\varepsilon}\right] & \longrightarrow\left(s_{\varepsilon}^{2}+\varepsilon^{2}\right) \partial_{t} \varphi_{\varepsilon} \quad \text { weakly in } L^{2}\left(\Omega_{T}\right) \\
\pi^{h}\left[\left(S_{\varepsilon}^{+}\right)^{2}+\varepsilon^{2}\right] \nabla \Phi_{\varepsilon}^{+} & \longrightarrow\left(s_{\varepsilon}^{2}+\varepsilon^{2}\right) \nabla \varphi_{\varepsilon} \quad \text { weak* in } L^{\infty}\left(0, T ;\left[L^{2}(\Omega)\right]^{d}\right)
\end{aligned}
$$

For any $\eta \in L^{2}\left(0, T ; C_{0}^{\infty}(\bar{\Omega})\right)$, we choose $\eta^{h} \equiv \pi^{h} \eta$ in (3.4b). On noting (3.14), (3.1), (3.7) and (3.5), we can pass to the limit $h, \tau \rightarrow 0$ in (3.4b) for the subsequence of (3.14) to obtain $(2.4 \mathrm{~b})$ for any $\eta \in L^{2}\left(0, T ; C_{0}^{\infty}(\bar{\Omega})\right)$. The desired result $(2.4 \mathrm{~b})$ for any $\eta \in L^{2}\left(0, T ; H_{0}^{1}(\Omega)\right)$, then follows from the denseness of $C_{0}^{\infty}(\bar{\Omega})$ in $H_{0}^{1}(\Omega)$.

In order to pass to the corresponding limit in $(3.4 \mathrm{a})$, we require some stronger convergence for the third term. Similarly to (2.22), we obtain from (3.6), (3.4b) with $\eta^{h}=\Phi_{\varepsilon}^{+}-\pi^{h} \phi,(3.13),(3.14),(3.1),(3.7)$ and (2.4b) with 
$\eta=\varphi_{\varepsilon}-\phi$ that

$$
\limsup _{h, \tau \rightarrow 0} \int_{\Omega_{T}}\left[\left(S_{\varepsilon}^{+}\right)^{2}+\varepsilon^{2}\right]\left|\nabla \Phi_{\varepsilon}^{+}\right|^{2} \mathrm{~d} x \mathrm{~d} t \leq \limsup _{h, \tau \rightarrow 0} \int_{\Omega_{T}} \pi^{h}\left[\left(S_{\varepsilon}^{+}\right)^{2}+\varepsilon^{2}\right]\left|\nabla \Phi_{\varepsilon}^{+}\right|^{2} \mathrm{~d} x \mathrm{~d} t=\int_{\Omega_{T}}\left(s_{\varepsilon}^{2}+\varepsilon^{2}\right)\left|\nabla \varphi_{\varepsilon}\right|^{2} \mathrm{~d} x \mathrm{~d} t .
$$

Similarly to (2.23)-(2.25), it follows from (3.13), (3.14), (3.15) and (3.12a,b) that on extracting a further subsequence that

$$
S_{\varepsilon}^{+} \nabla \Phi_{\varepsilon}^{ \pm} \longrightarrow s_{\varepsilon} \nabla \varphi_{\varepsilon} \quad \text { strongly in } L^{2}\left(0, T ;\left[L^{2}(\Omega)\right]^{d}\right) \text { as } h, \tau \rightarrow 0 .
$$

Let $v \in L^{2}\left(0, T ; C_{0}^{\infty}(\bar{\Omega})\right)$. If $\delta=0$, we choose $v^{h}=\pi^{h} v$ in (3.4a). It follows from (2.6), (3.11), (3.13), (3.16), (3.1), (3.7), (3.8) and (3.5) that we can pass to the limit $h, \tau \rightarrow 0$ for a subsequence of (3.4a) to obtain (2.4a) with $\delta=0$ for any $v \in L^{2}\left(0, T ; C_{0}^{\infty}(\bar{\Omega})\right)$.

In order to achieve the corresponding limit in the case $\delta>0$, we have to once again exploit "the decisive monotonicity trick". It follows from the monotonicity of $|a|^{p-2} a, a \in \mathbb{R}^{d},(3.4 \mathrm{a}),(2.6),(3.11)$ and $s_{\varepsilon} \in$ $L^{\infty}\left(0, T ; W^{1, p}(\Omega)\right)$ with $p>d$ that for all $\lambda \in \mathbb{R}$ and for all $v \in L^{2}\left((0, T) ; C_{0}^{\infty}(\bar{\Omega})\right)$

$$
\begin{aligned}
0 \leq k_{1} \int_{\Omega_{T}}\left\{\left[1+\delta\left|\nabla\left(s_{\varepsilon}+\lambda v\right)\right|^{p-2}\right] \nabla\left(s_{\varepsilon}+\lambda v\right)-\left[1+\delta\left|\nabla S_{\varepsilon}^{+}\right|^{p-2}\right] \nabla S_{\varepsilon}^{+}\right\} \cdot \nabla\left[s_{\varepsilon}+\lambda v-S_{\varepsilon}^{+}\right] \mathrm{d} x \mathrm{~d} t \\
=k_{1} \int_{\Omega_{T}}\left[1+\delta\left|\nabla\left(s_{\varepsilon}+\lambda v\right)\right|^{p-2}\right] \nabla\left(s_{\varepsilon}+\lambda v\right) \cdot \nabla\left[s_{\varepsilon}+\lambda v-S_{\varepsilon}^{+}\right] \mathrm{d} x \mathrm{~d} t \\
\quad+\int_{0}^{T}\left\{k_{2}\left(\left|\nabla \varphi_{\varepsilon, \tau}^{-}\right|^{2}, \pi^{h}\left[S_{\varepsilon}^{+}\left(s_{\varepsilon}+\lambda v-S_{\varepsilon}^{+}\right)\right]\right)+\left(\partial_{t} S_{\varepsilon}+W_{+}^{\prime}\left(S_{\varepsilon}^{+}\right)-W_{-}^{\prime}\left(S_{\varepsilon}^{-}\right), \pi^{h}\left[s_{\varepsilon}+\lambda v\right]-S_{\varepsilon}^{+}\right)^{h}\right\} \mathrm{d} t \\
\quad-k_{1} \int_{\Omega_{T}}\left[1+\delta\left|\nabla S_{\varepsilon}^{+}\right|^{p-2}\right] \nabla S_{\varepsilon}^{+} \cdot \nabla\left[\left(I-\pi^{h}\right)\left(s_{\varepsilon}+\lambda v\right)\right] \mathrm{d} x \mathrm{~d} t .
\end{aligned}
$$

It follows from (3.8), (3.11) and an inverse inequality that

$$
\int_{0}^{T}\left\|\left(I-\pi^{h}\right)\left[S_{\varepsilon}^{+}\left(\pi^{h}\left[s_{\varepsilon}+\lambda v\right]-S_{\varepsilon}^{+}\right)\right]\right\|_{L^{\infty}}^{2} \mathrm{~d} t \leq C h^{2\left(1-\frac{d}{p}\right)} \int_{0}^{T}\left\|\nabla\left(\pi^{h}\left[s_{\varepsilon}+\lambda v\right]-S_{\varepsilon}^{+}\right)\right\|_{L^{p}}^{2} \mathrm{~d} t .
$$

On noting (3.13) with $q_{1} \in[1, \infty],(3.1),(3.7),(3.5),(3.11),(3.16),(3.18)$ and as $p>d$, we now pass to the limit $h, \tau \rightarrow 0$ in a subsequence of (3.17) yielding for all $\lambda \in \mathbb{R}$ and for all $v \in L^{2}\left((0, T) ; C_{0}^{\infty}(\bar{\Omega})\right)$ that

$$
0 \leq \lambda \int_{\Omega_{T}}\left\{k_{1}\left[1+\delta\left|\nabla\left(s_{\varepsilon}+\lambda v\right)\right|^{p-2}\right] \nabla\left(s_{\varepsilon}+\lambda v\right) \cdot \nabla v+\left[\partial_{t} s_{\varepsilon}+k_{2} s_{\varepsilon}\left|\nabla \varphi_{\varepsilon}\right|^{2}+W^{\prime}\left(s_{\varepsilon}\right)\right] v\right\} \mathrm{d} x \mathrm{~d} t .
$$

Considering the cases of $\lambda>0$ and $\lambda<0$ separately, we divide (3.19) by $\lambda$ and then pass to the limit $\lambda \rightarrow 0$ to obtain, on combining with the previously derived $\delta=0$ case, the desired result (2.4a) for any $v \in L^{2}\left((0, T) ; C_{0}^{\infty}(\bar{\Omega})\right)$.

The desired result (2.4a) with $\delta \geq 0$ for any $v \in L^{2}\left(0, T ; W_{0}^{1, p^{\star}}(\Omega) \cap L^{\infty}(\Omega)\right)$ then follows from noting that $C_{0}^{\infty}(\bar{\Omega})$ is dense in $W_{0}^{1, p^{\star}}(\Omega) \cap L^{\infty}(\Omega)$, with the strong topology on $W_{0}^{1, p^{\star}}(\Omega)$ and the weak* topology on $L^{\infty}(\Omega)$, e.g. adapt the proof of Lemma 2.5 ([5], p. 119).

\subsection{Convergence of $\left(\mathbf{P}_{\delta, \varepsilon}^{h, \tau}\right)$ as $h, \tau, \varepsilon \rightarrow 0$}

Theorem 3.2. Let either (a) $\delta=0$ if $d=1$ or (b) $\delta>0$ and $p>d$ if $d \geq 1$. Then there exists a subsequence $\left\{S_{\varepsilon}, \Phi_{\varepsilon}\right\}_{h, \tau, \varepsilon>0}$ such that as $h, \tau, \varepsilon \rightarrow 0$

$$
S_{\varepsilon} \longrightarrow s \quad \text { strongly in } L^{q_{1}}\left(\Omega_{T}\right), \quad S_{\varepsilon} \Phi_{\varepsilon} \longrightarrow s \varphi \quad \text { strongly in } L^{q_{2}}\left(\Omega_{T}\right) ;
$$


where $\{s, \phi\}$ is a weak solution to $\left(P_{\delta}\right)$ and $q_{1} \in[1, \infty], q_{2} \in[1, \infty)$.

Proof. Combining Theorem 2.9, Theorem 3.1 and (2.45) yields the desired result.

\section{NumericAl EXPERIMENTS}

In this final section we present several numerical experiments in two space dimensions to gauge the fully discrete finite element method, $\left(\mathrm{P}_{0, \varepsilon}^{h, \tau}\right)$, developed in the previous section. In addition, our numerical results reveal some interesting features, such as existence, formation and annihilation of line singularities (defects), of the Ericksen model $\left(\mathrm{P}_{0}\right),(1.1 \mathrm{a}, \mathrm{b})$. In all the numerical experiments below, unless mentioned otherwise, we choose $\Omega=[0,1]^{2}, \delta=0, k_{1}=k_{2}=k_{3}=1$, and $W(s)=\frac{5}{2}\left(s^{2}-1\right)^{2}$, cf. [2]. Throughout, we use a uniform isoceles right-angled triangulation of $\Omega$ with $h=\sqrt{2} \widehat{h}$ and a uniform time step $\tau$.

We start with an academic test example to highlight the numerical difficulties arising from the degeneracy of the problem $\left(\mathrm{P}_{0}\right)$.

Test 1. Let $\left\{\mathcal{F}\left(x_{1}, x_{2}, t\right), \mathcal{G}\left(x_{1}, x_{2}, t\right)\right\}$ be chosen such that

$$
s\left(x_{1}, x_{2}, t\right)=4\left(x_{1}\left(1-x_{1}\right) x_{2}\left(1-x_{2}\right)\left(1+t^{2}\right)-0.1\right), \quad \varphi\left(x_{1}, x_{2}, t\right)=\frac{x_{1}^{2}}{2}\left(1+t^{2}\right)
$$

solve the problem

$$
\partial_{t} s-\Delta s+|\nabla \varphi|^{2} s+W^{\prime}(s)=\mathcal{F}, \quad s^{2} \partial_{t} \varphi-\operatorname{div}\left(s^{2} \nabla \varphi\right)=\mathcal{G} \quad \text { in } \Omega_{T} .
$$

We remark that in order to approximate the solution of $(4.2)$, the nonhomogeneous terms $\{\mathcal{F}, \mathcal{G}\}$ have to be added to the right-hand sides of the fully discrete scheme $(3.3 \mathrm{a}, \mathrm{b})$, and of the course the initial and boundary conditions need to be modified. Obviously these modifications destroy the dissipative energy structure and the maximum/minimum principles for $\left(\mathrm{P}_{0}\right),\left(\mathrm{P}_{0, \varepsilon}\right)$ and $\left(\mathrm{P}_{0, \varepsilon}^{h, \tau}\right)$. We note that $\left\{(x, t) \in \Omega_{T}: s(x, t)=0\right\}$ is not empty for $t \geq \sqrt{\frac{3}{5}}$ and hence the degeneracy in the system plays a crucial role.

We computed the unique numerical solution $\left\{S_{\varepsilon}, \Phi_{\varepsilon}\right\}$ to the modified scheme $\left(\mathrm{P}_{0, \varepsilon}^{h, \tau}\right)$, $(3.3 \mathrm{a}, \mathrm{b})$, with fixed $\widehat{h}=0.02$ and $\tau=0.0025$ with two different choices of $\varepsilon: 0.01$ and 0.11 . The first two rows of Figure 1 show the computed solution $\left\{\Phi_{\varepsilon}(\cdot, t), S_{\varepsilon}(\cdot, t)\right\}$ with $\varepsilon=10^{-2}$ at $t=0.75,1.125$ and 1.5625. A significant error in $\Phi_{\varepsilon}(\cdot, t)$ is clearly visible where $\left\{x \in \Omega: S_{\varepsilon}(x, t)=0\right\} \neq \emptyset$. However, this error is significantly reduced when the regularization parameter, $\varepsilon$, is increased to 0.11 . This can be seen from the bottom row of Figure 1 , where $\Phi_{\varepsilon}(\cdot, t)$ with $\varepsilon=0.11$ is plotted at $t=1.125,1.5$ and 1.5625 . The above observation suggests that the mesh sizes $h$ and $\tau$ should be reduced in line with the regularization parameter $\varepsilon$, otherwise, the accuracy of the approximation $\left\{S_{\varepsilon}, \Phi_{\varepsilon}\right\}$ is not guaranteed. For this problem we observed also (slow) strong convergence of $S_{\varepsilon}$ to $s$ in $L^{\infty}\left(0, T ; L^{2}(\Omega)\right)$, but no strong convergence of $\Phi_{\varepsilon}$ to $\varphi$ in $L^{\infty}\left(0, T ; L^{2}(\Omega)\right)$ as $h, \tau, \varepsilon \rightarrow 0$. This supports our convergence results in Subsection 3.3.

The system $\left(\mathrm{P}_{0}\right),(1.1 \mathrm{a}, \mathrm{b})$, is driven solely by the initial and boundary conditions on $s$ and $\varphi$. For the analysis in the previous sections, we chose the compatible initial/boundary data $(2.1, b)$. In order to illustrate the formation, annihilation and evolution of line singularities/defects in such a model, it is convenient to choose, in some cases, incompatible data.

Test 2. Throughout these experiments, we take

$$
\phi\left(x_{1}, x_{2}\right)= \begin{cases}\pi & \text { for } x_{1} \geq 0.5 \\ 0 & \text { otherwise }\end{cases}
$$

With this choice, the director field $\mathbf{n}:=(\cos (\varphi), \sin (\varphi))$ initially has a line singularity at $x_{1}=0.5$. Firstly, we take the following incompatible initial/boundary data for $s$

$$
s\left(x_{1}, x_{2}, 0\right)=\max \left\{x_{1}-0.5,0\right\} \quad \forall x \in \Omega, \quad s\left(x_{1}, x_{2}, t\right)=x_{1}-0.5 \quad \forall x \in \partial \Omega, \quad t>0 .
$$



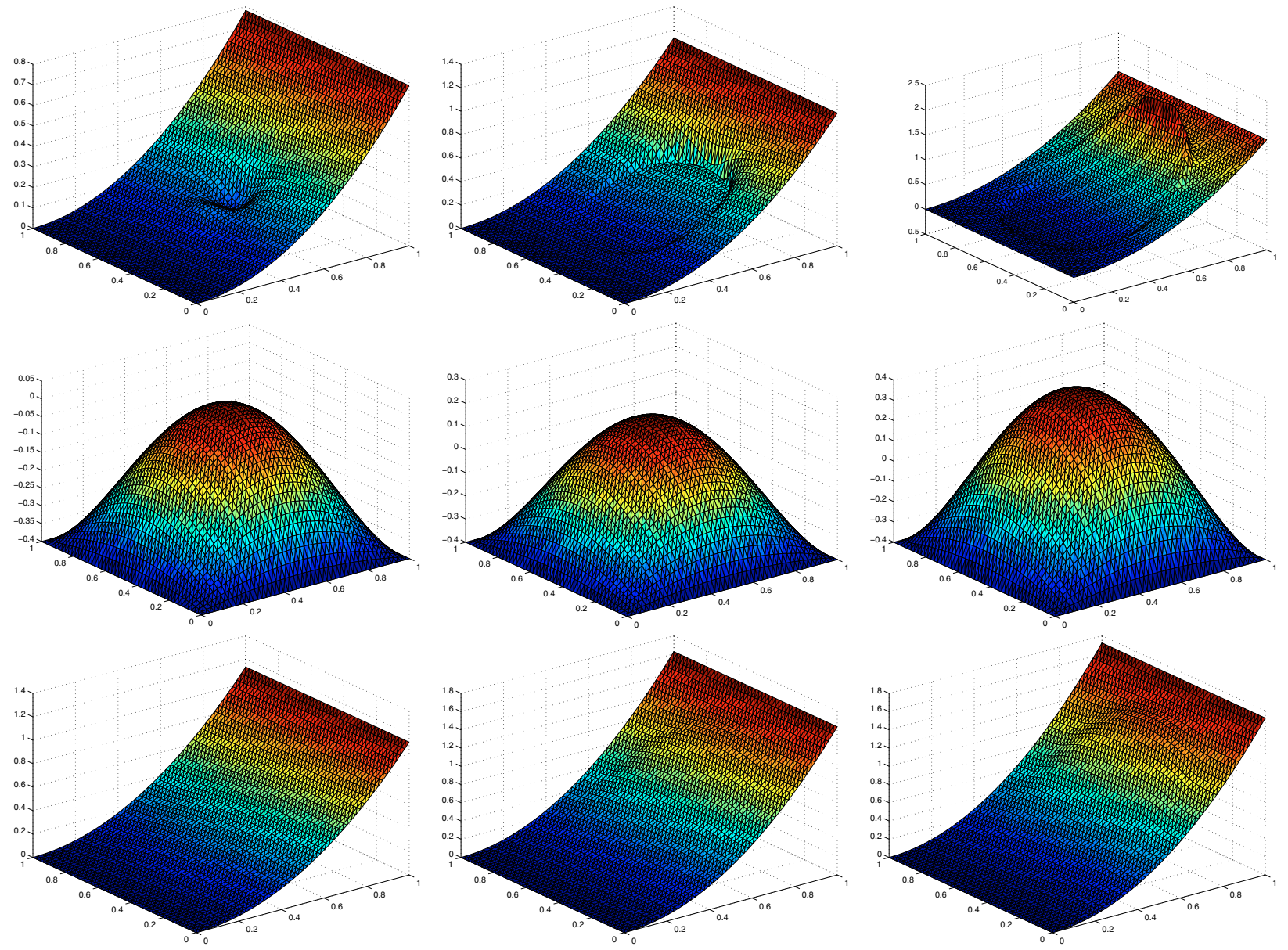

Figure 1. First and second rows: $\Phi_{\varepsilon}(\cdot, t)$ and $S_{\varepsilon}(\cdot, t)$ at $t=0.75,1.125$ and 1.5625 with $\varepsilon=0.01$. Third row: $\Phi_{\varepsilon}(\cdot, t)$ at $t=1.125,1.5$ and 1.5625 with $\varepsilon=0.11$.

We computed the unique numerical solution $\left\{S_{\varepsilon}, \Phi_{\varepsilon}\right\}$ to the modified scheme $\left(\mathrm{P}_{0, \varepsilon}^{h, \tau}\right)$ with $\widehat{h}=0.04, \tau=0.001$ and $\varepsilon=0.07$. The first two rows of Figure 2 show the computed director field $\mathbf{N}_{\varepsilon}(\cdot, t):=\left(\cos \left(\Phi_{\varepsilon}(\cdot, t)\right), \sin \left(\Phi_{\varepsilon}(\cdot, t)\right)\right)$ and $S_{\varepsilon}(\cdot, t)$ at $t=0.001,0.01$ and 0.048 . We see that the structure of the singularity for $\mathbf{N}_{\varepsilon}$ remains during the evolution, whereas $S_{\varepsilon}$ evolves to be, approximately, the linear profile $x_{1}-0.5$; which vanishes along the jump in $\Phi_{\varepsilon}$. This evolution can be expected from inspecting the dissipative energy law $(3.12 \mathrm{a}, \mathrm{b})$ and the boundary data in (4.4). Finally the third row of Figure 2 shows the rapid decay of the energy (3.12b) plotted against time levels (iterations) $m$.

Figures 3 and 4 contain similar snapshots of $\mathbf{N}_{\varepsilon}$ and $S_{\varepsilon}$ with exactly the same data as in Figure 2 except with a different choice of initial data for $s: s\left(x_{1}, x_{2}, 0\right)=0$ and $s\left(x_{1}, x_{2}, 0\right)=0.5$, respectively. Similar solution behaviors are observed as in Figure 2. There is also a similar energy decay in all the experiments below, but not necessarily displayed.

In the above simulations, the boundary data for $s$ vanishes at $x_{1}=0.5$, where $\phi$ has a jump. Figure 5 gives similar snapshots of $\mathbf{N}_{\varepsilon}$ and $S_{\varepsilon}$ with exactly the same data as in Figure 2 except $k_{1}=10$ and (4.4) replaced by

$$
s\left(x_{1}, x_{2}, 0\right)=0.5 \quad \forall x \in \Omega, \quad s\left(x_{1}, x_{2}, t\right)=1-x_{1} \quad \forall x \in \partial \Omega, \quad t>0 .
$$



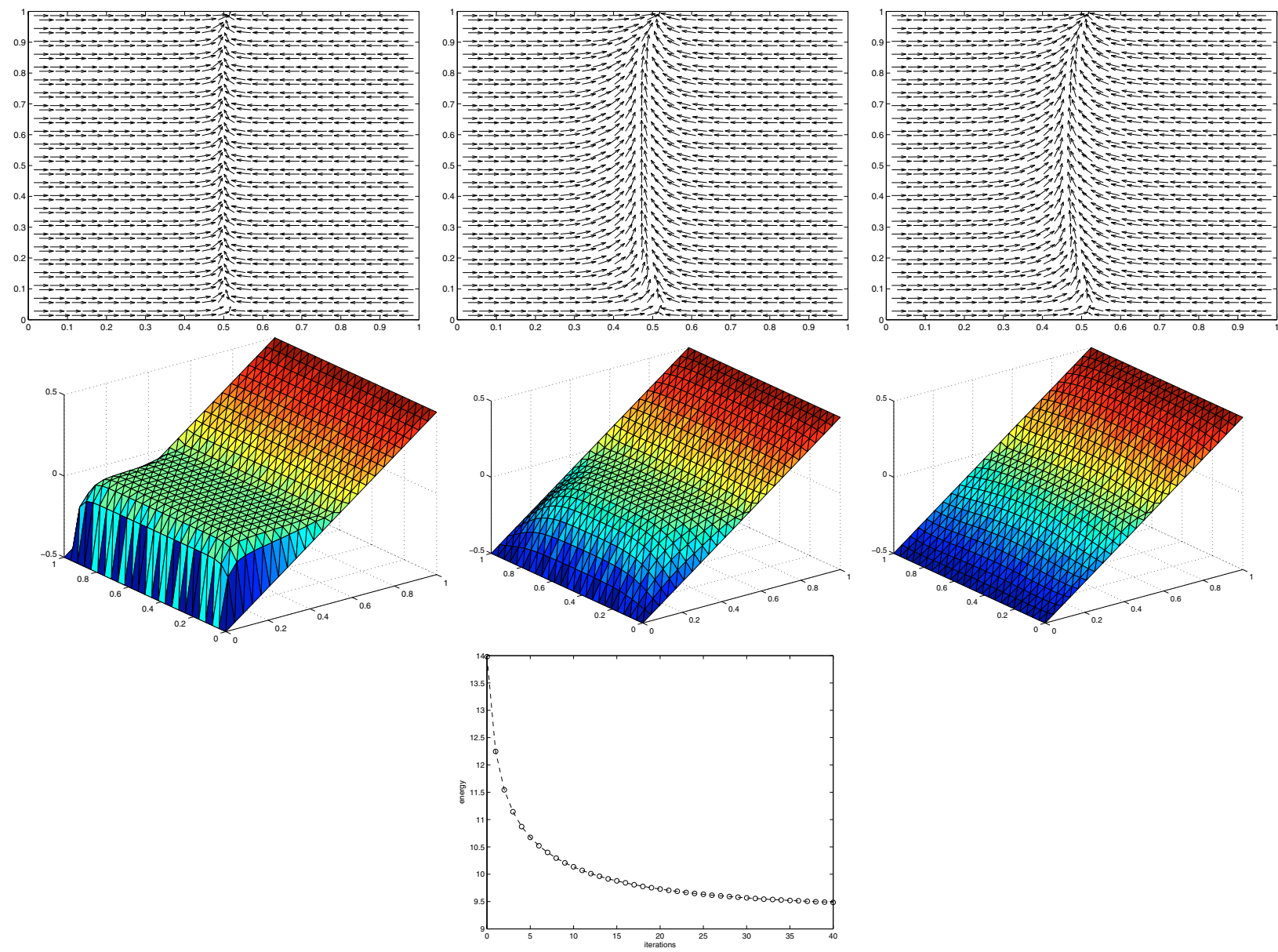

Figure 2. Stability of line defect at $\left\{x_{1}=0.5\right\}: \mathbf{N}_{\varepsilon}(\cdot, t)$ and $S_{\varepsilon}(\cdot, t)$ at $t=0.001,0.01$ and 0.048. Third row: Decay of the energy $(3.12 \mathrm{~b})$ during the evolution.

This simulation suggests that the initial line singularity in $\phi$ is not stable if $s$ vanishing at $\left\{x_{1}=0.5\right\}$ is not energetically attractive.

Test 3. Our third test shows an interesting situation in which two line singularities in n, with opposite orientations, cancel each other during the evolution. We chose

$$
g\left(x_{1}, x_{2}\right)=\left(x_{1}-0.3\right)\left(x_{1}-0.7\right), \quad \phi\left(x_{1}, x_{2}\right)= \begin{cases}\pi & \text { for } 0.3 \leq x_{1} \leq 0.7 \\ 0 & \text { otherwise }\end{cases}
$$

and $\widehat{h}=0.04, \tau=0.001$ and $\varepsilon=0.07$.

Figure 6 shows $\mathbf{N}_{\varepsilon}(\cdot, t)$ at $t=0.001,0.002,0.004$ and $0.015, S_{\varepsilon}(\cdot, 0.095)$ and the decay of the energy $(3.12 \mathrm{~b})$. Here we observe the annihilation of the initial line defects in $\mathbf{N}_{\varepsilon}$, but vortices appear at the boundary due to the imposed boundary condition on $\Phi_{\varepsilon}$. In addition $S_{\varepsilon}$ flattens out in the interior of $\Omega$, in order to reduce the contribution of $\left|\nabla S_{\varepsilon}\right|^{2}$ in the energy $(3.12 \mathrm{~b})$.

Figure 7 shows similar snapshots of $\mathbf{N}_{\varepsilon}$ with exactly the same data as in Figure 6 except with $\phi$ replaced by

$$
\phi\left(x_{1}, x_{2}\right)=\left\{\begin{aligned}
\frac{\pi}{2} & \text { for } 0.3 \leq x_{1} \leq 0.7 \\
-\frac{\pi}{2} & \text { otherwise }
\end{aligned}\right.
$$



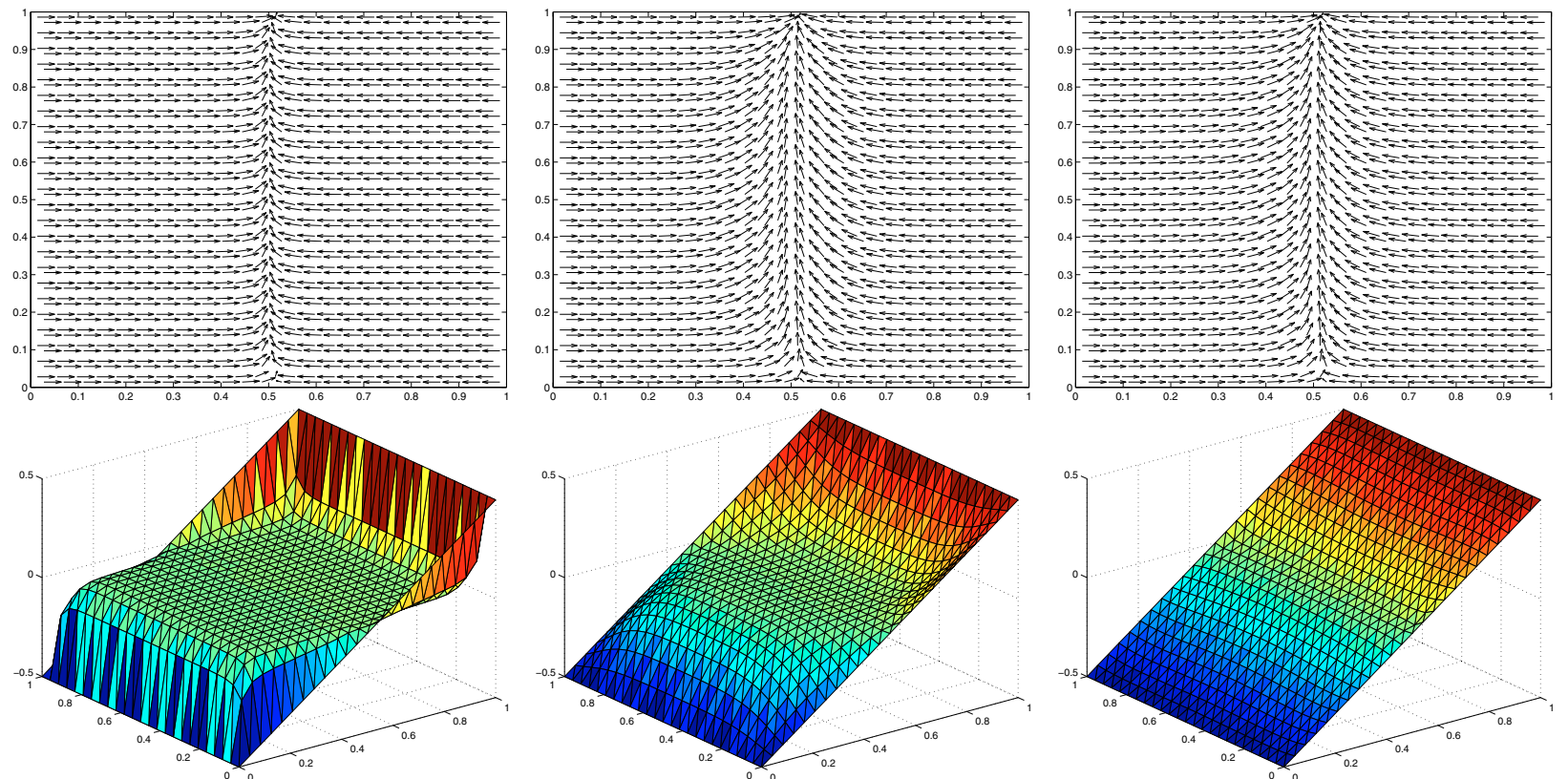

Figure 3. Stability of line defect at $\left\{x_{1}=0.5\right\}: \mathbf{N}_{\varepsilon}(\cdot, t)$ and $S_{\varepsilon}(\cdot, t)$ at $t=0.001,0.01$ and 0.05 .
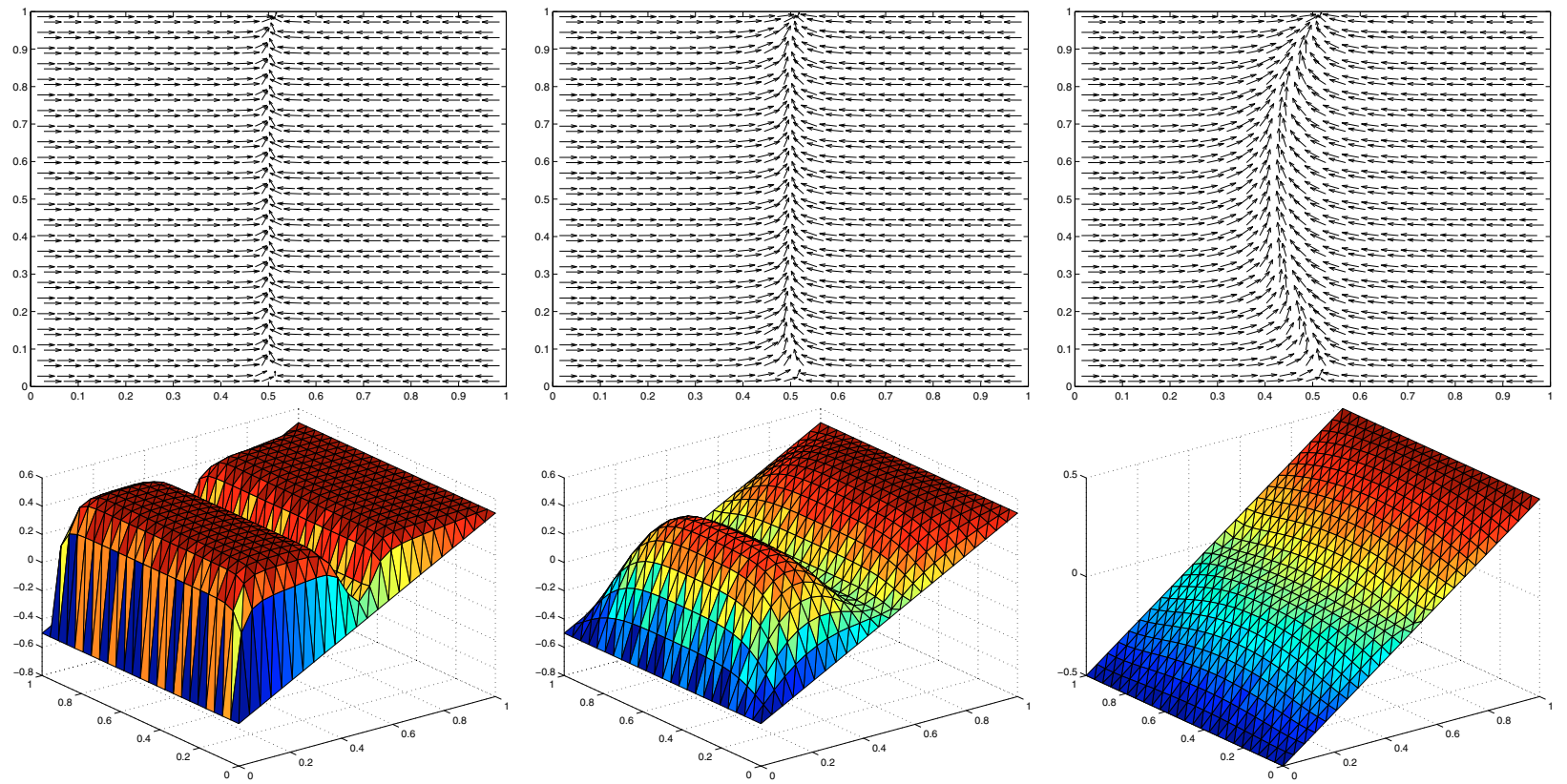

Figure 4. Stability of line defect at $\left\{x_{1}=0.5\right\}: \mathbf{N}_{\varepsilon}(\cdot, t)$ and $S_{\varepsilon}(\cdot, t)$ at $t=0.001,0.01$ and 0.048 .

If we keep the initial conditions of either of the previous experiments, but we replace the boundary conditions on $S_{\varepsilon}$ and $\Phi_{\varepsilon}$ by homogenous data; then of course $S_{\varepsilon}(\cdot, t) \rightarrow 0$ and $\mathbf{N}_{\varepsilon}(\cdot, 0) \rightarrow(1,0)$ as $t$ increases. 

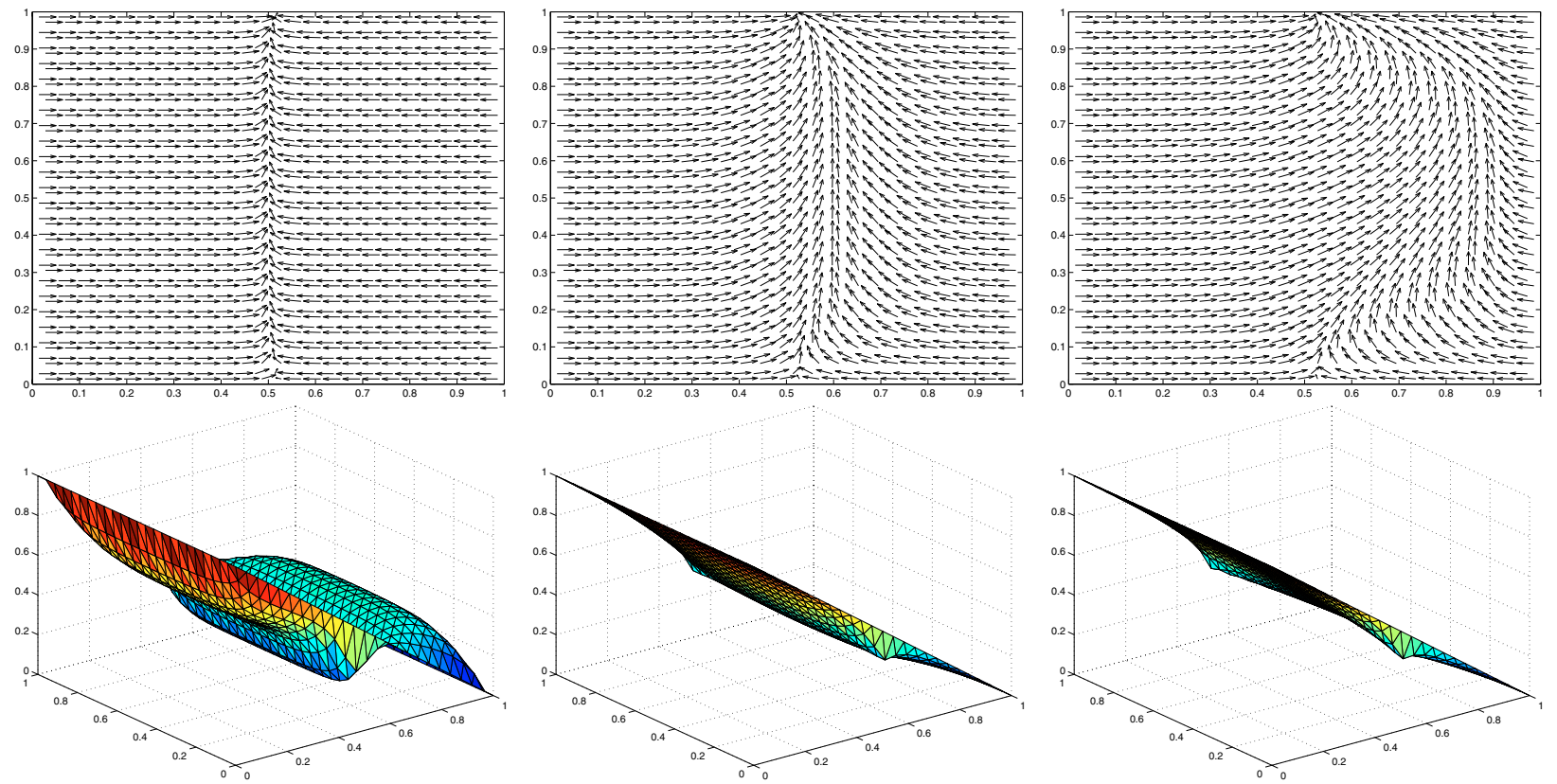

Figure 5. Instability of line defect at $\left\{x_{1}=0.5\right\}: \mathbf{N}_{\varepsilon}(\cdot, t)$ and $S_{\varepsilon}(\cdot, t)$ with $k_{1}=10$ at $t=0.001,0.020$ and 0.35 .
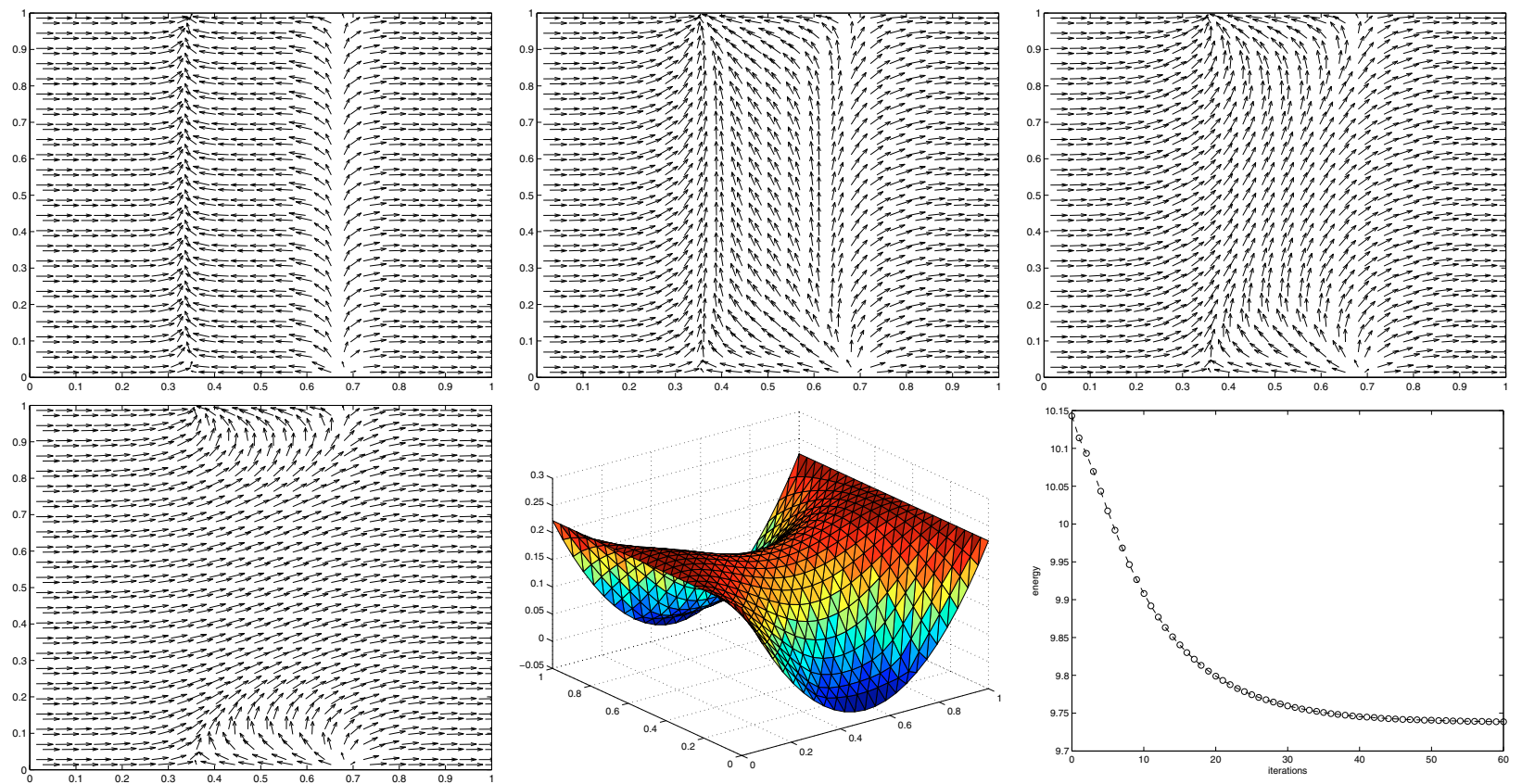

Figure 6. Annihilation of line defects: $\mathbf{N}_{\varepsilon}(\cdot, t)$ at $t=0.001,0.002,0.004$ and $0.015, S_{\varepsilon}(\cdot, 0.095)$ and the decay of the energy $(3.12 \mathrm{~b})$. 

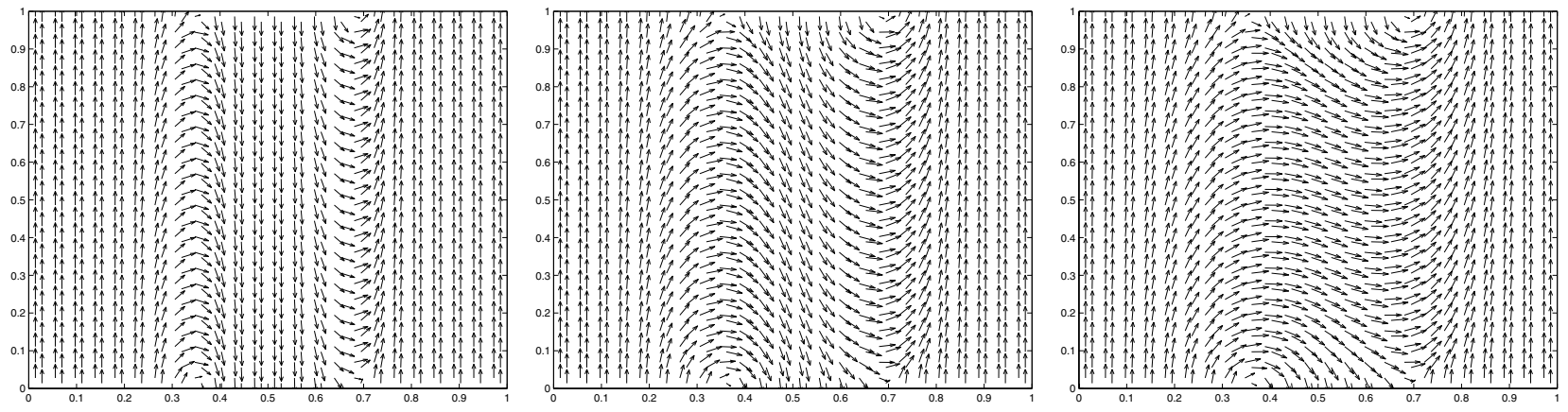

Figure 7. Annihilation of line defects: $\mathbf{N}_{\varepsilon}(\cdot, t)$ at $t=0.001,0.005$ and 0.02 .
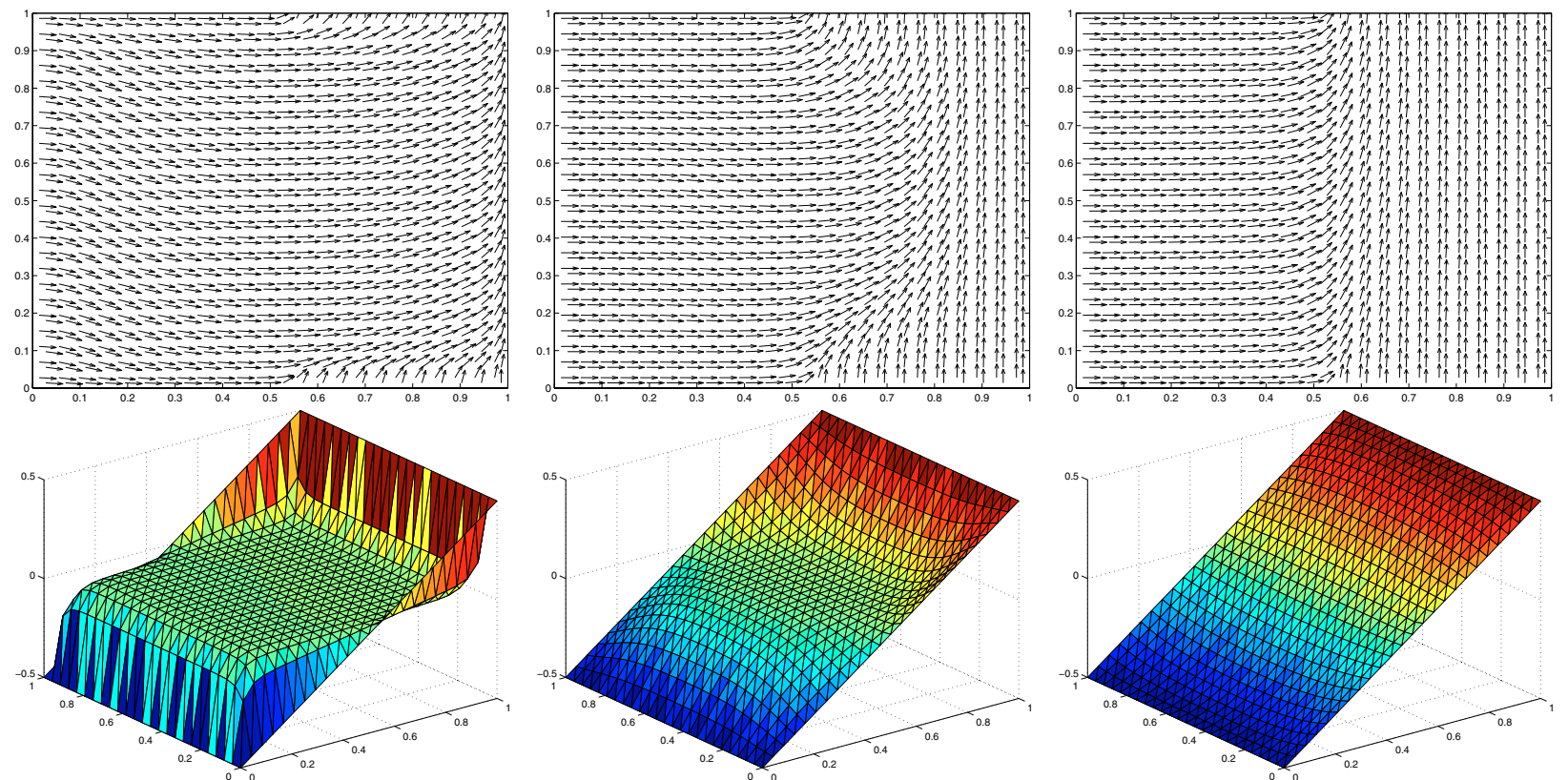

Figure 8. Generation of line defect at $\left\{x_{1}=0.5\right\}: \mathbf{N}_{\varepsilon}(\cdot, t)$ and $S_{\varepsilon}(\cdot, t)$ at $t=0.001,0.015$ and 0.18 .

Test 4. Our last test studies the generation of line singularities in $\mathbf{N}_{\varepsilon}$, in particular, for large times. We chose the following incompatible initial/boundary data for $s$ and $\varphi$

$$
\begin{array}{ll}
s\left(x_{1}, x_{2}, 0\right)=0, & \varphi\left(x_{1}, x_{2}, 0\right)=x_{1}-\frac{1}{2} ; \\
s(x, y, t)=x_{1}-\frac{1}{2} \quad \forall x \in \partial \Omega, t>0, \quad \varphi\left(x_{1}, x_{2}, t\right)= \begin{cases}0 & \text { for } x \in \partial \Omega, x_{1} \leq 0.5, t>0, \\
\frac{\pi}{2} & \text { for } x \in \partial \Omega, x_{1}>0.5, t>0 .\end{cases}
\end{array}
$$

We then computed our approximation $\left\{S_{\varepsilon}, \Phi_{\varepsilon}\right\}$ with $\widehat{h}=0.04, \tau=0.001$ and $\varepsilon=0.07$. Figure 8 shows $\left\{\mathbf{N}_{\varepsilon}(\cdot, t), S_{\varepsilon}(\cdot, t)\right\}$ at $t=0.001,0.015$ and 0.18 . A line singularity in $\mathbf{N}_{\varepsilon}$ is generated during the evolution and it stabilizes at $\left\{x_{1}=0.5\right\}$, which is the zero-level set of $S_{\varepsilon}$, for large $t$. 
Remark 4.1. The numerical computations in Tests $2-4$ above, showing the formation, annihilation and evolution of line singularities/defects in $\left(\mathrm{P}_{0, \varepsilon}^{h, \tau}\right)$, can be explained in terms of the energy decay of $E_{\varepsilon}^{h}(\cdot, \cdot)$, recall $(3.12 \mathrm{a}, \mathrm{b})$ with $\delta=0$; even though incompatible initial/boundary data is not directly covered by the theory in the previous sections. As noted previously, the energy structure for $\left(\mathrm{P}_{\delta, \varepsilon}^{h, \tau}\right)$ is very similar to that for $\left(\mathrm{P}_{0, \varepsilon}^{h, \tau}\right)$; and so it is not surprising that the corresponding numerical computations for $\left(\mathrm{P}_{\delta, \varepsilon}^{h, \tau}\right)$, with $p-2$ and $\delta$ small and positive, are graphically indistinguishable from those reported for $\left(\mathrm{P}_{0, \varepsilon}^{h, \tau}\right)$. For example in Tests 2 and 4 , $S_{\varepsilon}$ evolves towards a linear function satisfying the imposed linear boundary conditions, so as to minimize the effect of the $\nabla S_{\varepsilon}$ term in the energy $E_{\varepsilon}^{h}(\cdot, \cdot)$. Obviously, the difference between small positive $\delta$ and $\delta=0$ is negligible on such an evolution.

\section{REFERENCES}

[1] R.A. Adams, Sobolev Spaces. Academic Press, New York (1975).

[2] M.C. Calderer, D. Golovaty, F.-H. Lin and C. Liu, Time evolution of nematic liquid crystals with variable degree of orientation. SIAM J. Math. Anal. 33 (2002) 1033-1047.

[3] C.M. Elliott and S. Larsson, A finite element model for the time-dependent joule heating problem. Math. Comp. 64 (1995) $1433-1453$.

[4] J.L. Ericksen, Liquid crystals with variable degree of orientation. Arch. Ration. Mech. Anal. 113 (1991) 97-120.

[5] R. Glowinski, Numerical Methods for Nonlinear Variational Problems. Springer-Verlag, Berlin (1984).

[6] N.G. Meyers, An $L^{\mathrm{p}}$ estimate for the gradient of solutions of second order elliptic divergence equations. Ann. Scuola Norm. Sup. Pisa 17 (1963) 189-206.

[7] X. Xu, Existence for a model arising from the in situ vitrification process. J. Math. Anal. Appl. 271 (2002) 333-342.

[8] E. Zeidler, Nonlinear Functional Analysis and Its Applications, Vol. II/B. Springer, New York (1990). 